\title{
Pore-fluid effects on seismic waves in vertically fractured earth with orthotropic symmetry
}

\author{
James G. Berryman* \\ *Lawrence Berkeley National Laboratory, Earth Sciences Division, Berkeley, California \\ E-mail: JGBerryman@LBL.GOV
}

(June 4, 2010)

Running head: Pore fluids \& seismic waves

\begin{abstract}
For elastically non-interacting vertical-fracture sets at arbitrary orientation angles to each other, I present a detailed model in which the resulting anisotropic fractured medium generally has orthorhombic symmetry overall. Some of the analysis methods and ideas of Schoenberg are emphasized, together with their connections to other similarly motivated and conceptually related methods by Sayers and Kachanov, among others. Examples show how parallel vertical-fracture sets having HTI (horizontal transversely isotropic) symmetry transform into orthotropic fractured media if some subsets of the vertical fractures are misaligned with the others, and then the fractured system can have VTI (vertical transversely isotropic) symmetry if all the fractures are aligned either randomly, or half parallel and half perpendicular to a given vertical plane. I compare and contrast an orthotropic example having vertical fractures in an otherwise VTI earth system (studied previously by Schoenberg and Helbig) with the other examples treated, and finally show how fluids in the fractures affect the orthotropic poroelastic system response to seismic waves. The key result is that fracture-influence parameters are multiplied by a factor of $(1-B)$, where $0 \leq B<1$
\end{abstract}


is Skempton's second coefficient for poroelastic media. Skempton's B coefficient is itself a measurable characteristic of fluid-saturated porous rocks, depending on porosity, solid moduli, and pore-fluid bulk modulus. For heterogeneous porous media, connections between the present work and earlier related results of Brown and Korringa are also established. 


\section{INTRODUCTION}

The present work treats various issues related to fractures and anisotropy, especially in relation to some of the published work of Michael Schoenberg (Schoenberg, 1980; Schoenberg and Muir, 1989; Schoenberg and Sayers, 1995; Schoenberg and Helbig, 1997). Details of methods presented here will also make use of an approach outlined by Sayers and Kachanov (1991), and used previously by Berryman $(2007,2008,2010)$ in a recent series of published papers. In earlier work, it has also been shown (Berryman, 2007) that the general results of (for example) Bakulin et al. (2000) for the Thomsen (1986, 2002) weak-anisotropy seismic parameters, and contained in their Figure 6, are both qualitatively and even (reasonably) quantitatively consistent with each other, as well as being consistent with results from the method of Sayers and Kachanov (1991) being treated explicitly. Thus, a high degreee of consistency is established among fracture-influence results that are based in part on the linear-slip model of fractures by Schoenberg (1980) and in other examples on penny-shaped (or approximately ellipsoidal) cracks. [Also see Grechka et al. (2006).] These relationships are important to the main theme of the paper, because they show that the details are often less important than the grand scheme of how fractures affect both the elastic-poroelastic system response and the seismic wave propagation results.

After establishing these main ideas, I will then change focus and show how fluids in the fractures alter the seismic wave speeds, by increasing the poroelastic stiffnesses. as well as the inertial density. While the present analysis is conceptually entirely consistent with earlier work on poroelasticity including that of Rice and Cleary (1976) and Cheng (1997), among many others, my emphasis differs somewhat since the preferred application is to seismic wave propagation problems rather than geomechanical analysis of reservoirs 
and hydrology. Also, there has been an effort made to avoid tensor notation (as much as possible) in this presentation, since our purpose is mostly to understand the effects of elastic/poroelastic responses on seismic waves, and this goal can be achieved rather easily without adding another level of mathematical sophistication to the problem.

My main poroelastic result shows that, for orthotropic media [but also see Sayers et al. (2009)], certain fracture-influence parameters (those pertinent to the principal stresses) are modified by a factor $(1-B)$, where $B$ is Skempton's second coefficient (Skempton, 1954).

Appendix A gives an extended discussion of pertinent results in poroelasticity for homogeneous porous media, while Appendix B compares and contrasts present methods with the work of Brown and Korringa (1975) on poroelastic materials having heterogeneous solid constituents.

\section{FRACTURE ANALYSIS}

For seismic waves propagating in the $\left[x_{1}-x_{3}\right]$-plane with wavenumbers $k_{1}=k \sin \theta$ and $k_{3}=k \cos \theta$ where $k^{2}=k_{1}^{2}+k_{3}^{2}$, Tsvankin (1997) shows that the following equations [patterned here after the notation of Berryman (1979)] are valid:

$$
\rho_{0} \omega_{ \pm}^{2}=\frac{1}{2}\left[\left(C_{11}+C_{55}\right) k_{1}^{2}+\left(C_{33}+C_{55}\right) k_{3}^{2} \pm \mathcal{R}\right]
$$

where

$$
\mathcal{R} \equiv \sqrt{\left[\left(C_{11}-C_{55}\right) k_{1}^{2}-\left(C_{33}-C_{55}\right) k_{3}^{2}\right]^{2}+4\left(C_{13}+C_{55}\right)^{2} k_{1}^{2} k_{3}^{2}}
$$

The inertial density is $\rho_{0}$ (with zero subscript, since $\rho_{f}$ is used later for fracture density). Equation (1) determines the two wave speeds

$$
V_{ \pm}^{2}=\frac{\omega_{ \pm}^{2}}{k^{2}}
$$


For the wave propagation studies of interest here, these $C_{i j}$ 's for $i, j=1, \ldots, 6$ are the elastic stiffnesses of the system of interest. [When the system is fractured, but no fluids (except air) are present in the fractures, I will talk later in the paper about the system being "drained." If the fractures are instead fluid-filled, then the notation will change to $S^{u}$ and $C^{u}$ for this "undrained" case. For present purposes, I need not make this distinction, as the mathematics for wave propagation is the same in either case.] The quantities $\omega_{ \pm}$ have dimensions of angular frequency, and are introduced mostly to simplify the form of the equations. The pertinent phase speeds $-V_{+}$for quasi- $P$-waves and $V_{-}$for quasi- $S V$-waves - are given respectively by values corresponding to the + and - subscripts in the velocity equation (3). Group velocities [Brillouin (1946); Ruger (2002); Tsvankin (2005)] can be computed routinely as well when using the methods outlined earlier by Berryman (1979).

This set of equations describes only one of three sets of equations that are valid for the orthotropic system to be studied here. The other two versions are obtained by permuting the indices, according to: $1 \rightarrow 2 \rightarrow 3 \rightarrow 1$, which corresponds to: $\left[x_{1}-x_{3}\right] \rightarrow$ $\left[x_{2}-x_{1}\right] \rightarrow\left[x_{3}-x_{2}\right]$. Then we have: $C_{11}, C_{33}, C_{13}, C_{55}, k_{1}, k_{3} \rightarrow C_{22}, C_{11}, C_{12}, C_{66}, k_{2}, k_{1} \rightarrow$ $C_{33}, C_{22}, C_{23}, C_{44}, k_{3}, k_{2}$. Each of the three versions behaves on its own much like a transversely isotropic (TI) or polar (i.e., having one preferred axis of symmetry) wave propagation system. It will therefore be sufficient to study this particular case using just equations (1) and (2) in order to understand the behavior of the more general problem.

Sayers and Kachanov (1991) consider a model with two sets of possibly nonorthogonal and noninteracting fractures, while also possibly having two different fracture density values $\rho_{a}$ and $\rho_{b}$. Fracture density can be defined (Bristow, 1960; Budiansky and O'Connell, 1976; Berryman and Aydin, 2010) to account for the well-known fact that the actual thickness (or aspect ratio $\alpha$ ) for thin fractures does not strongly control their influence on the mechanics 
of a fractured medium. Similarly, the porosity $\phi$ associated with a fracture or set of fractures is also not a pertinent measure of fracture influence since it is proportional to the fracture aspect ratio. A better measure of the crack/fracture influence is therefore given instead by the ratio

$$
\rho_{f} \equiv \phi / \alpha
$$

assuming that the cracks all have approximately the same aspect ratio $\simeq \alpha$. If that is not the case, then $\alpha$ should be replaced with an appropriately averaged value $\langle\alpha\rangle$. Alternative definitions may be found in the references. When there are two distinct types of fractures present, the total fracture density is given by the sum $\rho_{f}=\rho_{a}+\rho_{b}$, which is the rule used by Sayers and Kachanov (1991). When the angle between the fracture sets is $\Phi$, Sayers and Kachanov (1991) found that the pertinent fracture-influence parameters were multiplied either solely by $\rho_{f}$ (total crack density) itself, or by one of the following two factors:

$$
\begin{aligned}
& \mathcal{A}_{f}=\rho_{f}+\left[\rho_{f}^{2}-4 \rho_{a} \rho_{b} \sin ^{2} \Phi\right]^{1 / 2}, \\
& \mathcal{B}_{f}=\rho_{f}-\left[\rho_{f}^{2}-4 \rho_{a} \rho_{b} \sin ^{2} \Phi\right]^{1 / 2} .
\end{aligned}
$$

In order to make use of these crack density factors, we also need to know the two fractureinfluence parameters $\eta_{1}$ and $\eta_{2}$. These results can be found in many places, including Sayers and Kachanov (1991), Bažant and Planas (1998), and Berryman and Grechka (2006). We therefore have formulas pertinent in the elastic non-interaction approximation (NIA) given by:

$$
\eta_{1}=-\frac{4 \nu_{0}\left(1-\nu_{0}\right)}{15 G_{0}\left(2-\nu_{0}\right)}
$$

and

$$
\eta_{2}=\frac{8\left(1-\nu_{0}\right)\left(5-\nu_{0}\right)}{15 G_{0}\left(2-\nu_{0}\right)}
$$

where $G_{0}$ is shear modulus for the unfractured background material, and $\nu_{0}$ is Poisson's ratio for the same material. When I say the formulas are pertinent for the non-interaction 
approximation, I mean that these simpler results apply only when the fractures do not interact mechanically among themselves. If there is mechanical interaction, then more complex effective-medium-style results are needed, and that is another story well beyond our current scope. Also, by way of clarification, it is important to realize that there is no assumption made here about hydraulic interaction. Mechanical non-interaction means that the fracture mechanics is being treated as if the cracks do not intersect each other; however, this does not imply that there might not be other means of hydraulic interaction via (for example) a double porosity mechanism in which the background material is itself porous and the fractures can therefore interact hydraulically through the pores of the host medium. It is also surely worth noting that, since $\nu_{0}$ typically lies in the range $0 \leq \nu_{0} \leq 0.5$, so then we have $\left|\eta_{1} / \eta_{2}\right| \leq 0.05$. This fact shows that $\eta_{1}$ usually produces less than a $5 \%$ perturbation to the overall quantitative predictions and, therefore, implies that we can often neglect $\eta_{1}$ altogether in field situations.

TABLE 1 shows the Sayers and Kachanov (1991) results for corrections to the isotropic background values of compliance (translated here into Voigt $6 \times 6$ matrix notation - results of the original paper being expressed instead in terms of tensor notation). Those background values are specifically for a model having effective bulk modulus $K_{0}=16.87$, shear modulus $G_{0}=2.20$, which then corresponds to Poisson's ratio $\nu_{0}=0.4375$ (dimensionless), and Young's modulus $E_{0}=6.325$, with all moduli (except Poisson's ratio) measured in units of GPa. For the assumed inertial density $\rho_{0}=2200.0 \mathrm{~kg} / \mathrm{m}^{3}$, the resulting isotropic background compressional wave speed is $V_{p}=3.0 \mathrm{~km} / \mathrm{s}$ and shear wave speed is $V_{s}=1.0 \mathrm{~km} / \mathrm{s}$. For these computations, I also need the isotropic background compliance values, which are $S_{11}=S_{22}=S_{33}=\frac{1}{E_{0}}=6.325, S_{12}=S_{13}=S_{23}=-\frac{\nu_{0}}{E_{0}}=-2.767$, and $S_{44}=S_{55}=S_{66}=\frac{1}{G_{0}}=0.4545$. The fracture influence factors $\eta_{1}$ and $\eta_{2}$, found for this spe- 
cific model by Berryman and Grechka (2006), are displayed in TABLE 2. Some higher order fracture-influence factors (namely, $\eta_{3}, \eta_{4}$, and $\eta_{5}$ ) were also determined in that earlier work, but I will not be considering such factors here, since they correspond to results important only for higher fracture densities that are beyond the scope of our current studies.

Figure 1 illustrates the general features of the conceptual model being considered here.

In the examples that follow, I will consider only the case of equal fracture densities $\rho_{a}=\rho_{b}=\rho_{f} / 2$. This simplified situation being somewhat easier to present both graphically and analytically, I will also be able to use the simplified factors:

$$
\begin{aligned}
& \mathcal{A}_{f}=\rho_{f}(1+\cos \Phi), \\
& \mathcal{B}_{f}=\rho_{f}(1-\cos \Phi),
\end{aligned}
$$

which follow directly from (5). There may also be some slight uncertainty about exactly which of these factors is which in this degenerate case, because of the sign ambiguity in taking the square root of $\cos ^{2} \Phi$. However, this detail will in no way affect the main quantitative results.

\section{VANISHING OF ANELLIPTICITY PARAMETERS}

One observation made immediately upon computing example velocities for the model specified here is that the quasi- $S V$-wave propagating in the $\left[x_{1}-x_{3}\right]$-plane using (1) and (2) apparently has constant (or very nearly constant to numerical accuracy) wave speed at all angles in this plane (see TABLE 3). This result is startling when first seen, but the general validity of the result has been remarked upon previously in the literature by Gassmann (1964) and also by Schoenberg and Sayers (1995). One useful interpretation of this fact is obtained by noting that, for quasi- $S V$-waves to have constant velocity in the plane, it 
is necessary for the pertinent effective anellipticity parameter to vanish for this plane of propagation. This parameter is given by

$$
\epsilon-\delta=\frac{X}{2 C_{33}\left(C_{33}-C_{55}\right)}
$$

where

$$
X \equiv\left(C_{11}+C_{13}\right)\left(C_{33}-C_{55}\right)-\left(C_{13}+C_{55}\right)\left(C_{33}+C_{13}\right)
$$

Here $\epsilon \equiv\left(C_{11}-C_{33}\right) / 2 C_{33}$ and $\delta \equiv\left[\left(C_{13}+C_{55}\right)^{2}-\left(C_{33}-C_{55}\right)^{2}\right] / 2 C_{33}\left(C_{33}-C_{55}\right)$ are two of Thomsen's three weak anisotropy coefficients. If the anellipticity parameter does not vanish identically, then it must at least vanish to first order in the fracture dependent correction factors (shown here in TABLE 1) in order to explain the numerical results for the relatively small fracture densities considered. Vanishing of (9) was shown earlier to be true in cases of low crack densities (Berryman, 2007).

The condition required for exact vanishing of the pertinent anellipticity parameter in terms of stiffness coefficients is:

$$
C_{11} C_{33}-C_{13}^{2}=C_{55}\left(C_{11}+C_{33}+2 C_{13}\right)
$$

which was already known to Gassmann (1964). This fact follows easily from (9) and (10), since the definition of $X$ can be rearranged into the form:

$$
X=C_{11} C_{33}-C_{13}^{2}-C_{55}\left(C_{11}+C_{33}+2 C_{13}\right)
$$

which clearly vanishes when (11) holds. The reason for this result is seen easily by a rearrangement of $(2)$

$$
\mathcal{R}=\sqrt{\left[\left(C_{11}-C_{55}\right) k_{1}^{2}+\left(C_{33}-C_{55}\right) k_{3}^{2}\right]^{2}+X k_{1}^{2} k_{3}^{2}} .
$$

It is easily seen that, if $X$ vanishes in (13), then both the velocities determined by (1) have simple elliptical symmetry, and no anelliptical contributions. 
Going farther in the analysis, it takes a fair amount of algebra to show — to first order in the correction factors - that the result (11) also amounts to a condition relating the compliance correction factors:

$$
\Delta S_{55}=\Delta S_{11}+\Delta S_{33}-2 \Delta S_{13}
$$

This condition holds when it is valid to ignore higher order terms such as $O\left(\Delta^{2}\right)$, plus all still higher orders (think of the proportionality $\Delta \propto \rho_{f}$, with $\rho_{f}$ a small number less than unity). Then, Equation (14) is satisfied identically when the expressions in TABLE 1 are substituted. It is important to notice as well that exact satisfaction of the condition in (14) is true for the general form of the definitions in TABLE 1, and not just for the restricted definitions used in the examples computed. The simplified definitions of (8) were employed to reduce our bookkeeping load. So the formula in (14) is more general than just the specific examples I have computed. The result is certainly not expected to be true for arbitrary compliance matrices. Nevertheless, it does turn out to be true for a wide range of compliance matrices having vertical fractures in an otherwise isotropic (or TI in the next section) earth body.

This result is already very interesting — maybe even surprising — but the curiously symmetric nature of this fracture model can be highlighted more fully by considering the following two expressions analogous (via permutation) to (14):

$$
\Delta S_{44}=\Delta S_{22}+\Delta S_{33}-2 \Delta S_{23}
$$

and

$$
\Delta S_{66}=\Delta S_{11}+\Delta S_{22}-2 \Delta S_{12}
$$

These two expressions are again both satisfied identically by the same set of expressions found in TABLE 1. These facts indicate that the model also has vanishing anellipticity 
factors (at least to the precision at which I am working) in the other orthogonal planes of propagation $\left[x_{2}-x_{3}\right]$ and $\left[x_{1}-x_{2}\right]$ as well.

The plane $\left[x_{1}-x_{2}\right]$, corresponding to the result (16), is not particularly interesting for exploration studies, since this is the horizontal plane, but the plane $\left[x_{2}-x_{3}\right]$ is of some additional interest. We can find the equations for this plane by taking the permutation $C_{11} \rightarrow C_{22}, C_{55} \rightarrow C_{44}$, and $C_{13} \rightarrow C_{23}$. Then, Eqs. (1) and (2) become:

$$
\rho_{0} \omega_{ \pm}^{2}=\frac{1}{2}\left[\left(C_{22}+C_{44}\right) k_{1}^{2}+\left(C_{33}+C_{44}\right) k_{3}^{2} \pm \mathcal{R}_{2}\right]
$$

where

$$
\begin{aligned}
\mathcal{R}_{2} & \equiv \sqrt{\left[\left(C_{22}-C_{44}\right) k_{1}^{2}-\left(C_{33}-C_{44}\right) k_{3}^{2}\right]^{2}+4\left(C_{23}+C_{44}\right)^{2} k_{1}^{2} k_{3}^{2}} \\
& \equiv \sqrt{\left[\left(C_{22}-C_{44}\right) k_{1}^{2}+\left(C_{33}-C_{44}\right) k_{3}^{2}\right]^{2}+Y k_{1}^{2} k_{3}^{2}},
\end{aligned}
$$

where

$$
Y \equiv C_{22} C_{33}-C_{23}^{2}-C_{44}\left(C_{22}+C_{33}+2 C_{23}\right)
$$

Clearly, the condition for vanishing anellipticity in this case is just $Y \equiv 0$, which is also equivalent (again to lowest order) to the condition (15).

As was known to Gassmann (1964), vanishing of the anellipticity factors means that there will be no triplications of wave arrivals for these models from propagation in any of these planes considered. Triplications arise because of complications from taking the partial derivatives required to compute group velocity from phase velocities quoted here [also see Berryman (1979) for examples]. Group velocity determines the wave speed of signals and/or pulses of seismic energy [see Brillouin (1946) for detailed discussion]. 


\section{VERTICAL FRACTURES IN VTI EARTH}

Another model in a closely related context that has been discussed frequently by Schoenberg and Helbig (1997), Bakulin (2000), and others is the model of a VTI earth system [where the background elastic medium is transversely isotropic with vertical axis of the system, as would occur in a layered-earth model having isotropic layers (Backus, 1962) with superposed vertical fractures. A result that is often quoted in this context concerns a condition (Schoenberg and Helbig, 1997) that is necessarily satisfied by the elastic stiffness matrix elements for such a system:

$$
C_{13}\left(C_{22}+C_{12}\right)=C_{23}\left(C_{11}+C_{12}\right)
$$

Using the same ideas applied already, this equation reduces to a simple statement about the system compliances.

The resulting formula connecting two off-diagonal compliances is:

$$
S_{13}=S_{23}
$$

by which it is meant that the only requirement imposed on the compliances after the introduction of the vertical fractures to the VTI earth background is that the new overall system compliance must satisfy the conditions in (21) after the changes due to the fractures are included in the values of these two compliance components. No other special constraints appear. To see that (21) is the correct condition, note that

$$
\begin{aligned}
& S_{13}=\left(C_{12} C_{23}-C_{13} C_{22}\right) / \operatorname{det}(C), \\
& S_{23}=\left(C_{12} C_{13}-C_{23} C_{11}\right) / \operatorname{det}(C),
\end{aligned}
$$

where $\operatorname{det}(C)$ is the determinant of the upper left $3 \times 3$ sub-matrix of the orthotropic stiffness matrix $C$. Equating these two expressions from (22) and rearranging the final result gives a formula that is precisely the same condition (20) found by Schoenberg and Helbig (1997) 
What this condition means for the physical system — since the background earth medium is assumed to be VTI with vertical axis of symmetry and also since $S_{13}=S_{23}$ for this background medium itself (before the fractures are added to it) - is that the final result for the overall compliance changes must therefore be the condition:

$$
\Delta S_{13}=\Delta S_{23}
$$

This very simple equality means that the changes (i.e., increases) in these off-diagonal compliances — being caused by the addition of the vertical fractures to this model have just the one constraint, and that constraint is on changes in these two off-diagonal compliances $S_{13}$ and $S_{23}$, which must occur in unison.

This result is also seen as a limiting case found in TABLE 1 , when $\mathcal{A}_{f}=\mathcal{B}_{f}$, which occurs only when $\Phi=0^{\circ}$. Since $\Phi=0^{\circ}$ means that all the vertical fractures are parallel, and therefore being aligned fractures, I have just recovered — using the Sayers and Kachanov (1991) approach — exactly the case studied, as well as the same results found, by Schoenberg and Helbig (1997).

\section{DISCUSSION OF VARIOUS FRACTURE-INFLUENCE MODELS}

Among others, there are two closely (though perhaps not obviously) related methods that are typically used in the seismic exploration literature for modeling the effects of fractures on seismic wave propagation: One method is the linear-slip-interface model introduced originally by Schoenberg (1980), while the other is a continuum approach that was introduced at about the same time by Kachanov (1980). These two methods have both been used extensively in the exploration community, especially since the work of Sayers and Kachanov (1991), Schoenberg and Sayers (1995), and Sayers and Kachanov (1995). Connections, in- 
cluding many similarities and a few differences, are discussed in the overview by Schoenberg and Sayers (1995). I have some personal preference for the analytical version of Sayers and Kachanov (1991), because it is so explicit while also permitting deep connections to be made directly to effective medium theories (Berryman, 2007), by taking advantage of results of Eshelby (1957). In particular, some recent and related work on fractures in outcrops using an effective medium theory approach by Berryman and Aydin (2010) has made use of the approach originally put forward by Sayers and Kachanov (1991) for modeling higher fracture density media. This study might have been more difficult to carry through using the original linear-slip-interface approach of Schoenberg (1980). But, once these higher fracture density results are known, it also becomes straightforward to incorporate them into the layer-averaging approach originated by Backus (1962) and later emphasized — as well as being reformulated for mathematical clarity — by Schoenberg and Muir (1989). Thus, these effective medium theories for layering were found to be very useful in providing a means of determining fracture-fracture interactions when the fracture sets are close, and therefore more dense, but not actually intersecting.

Figures 2, 3, and 4 illustrate the general behavior of seismic wave speeds for such models of vertical fractures in either homogeneous, isotropic earth or in VTI (vertical transversely isotropic) earth models with vertical fractures. Figure 2 shows quasi-compressional wave speed behavior, while Figures 3 and 4 display quasi-SV waves and SH-wave speeds, respectively.

Effects of fluids on the fracture behavior have not been emphasized here so far, but this issue is obviously a very important one for our applications, and it has been treated in other recent work by Daley et al. (2006) and Berryman (2007). Liquid effects on elastic moduli may be incorporated fairly easily using results of Gassmann (1951a,b) and Skempton 
(1954), as was also shown recently by Berryman (2007). The following section will treat these issues in more detail for the anisotropic systems being studied. Our main result will be to show that the behavior for fractured poroelastic systems is qualitatively the same as for fractured elastic systems, but the fluid influences the wave speeds by stiffening the fractures via a uniform factor (depending only on Skempton's second coefficient $B$ ).

\section{ANISOTROPIC POROELASTICITY}

Now the discussion will focus on a particular formulation of the equation of poroelasticity. Emphasis is placed on the compliance form of these equations. Clearly, the physics does not change when we go back and forth between compliance and stiffness forms of these equations. But the ease of interpretation is greatly enhanced in the compliance form. And, because we are treating fractures, which themselves directly introduce increases in elastic compliance, the formulation is also good for that reason as well.

If the overall porous medium is anisotropic due either to some preferential alignment of the constituent (anisotropic) particles or due to externally imposed stress (such as a gravity field and weight of overburden on constituent grains), for example, or due to aligned fractures, I can in all these situations consider the orthorhombic anisotropic version of the poroelastic equations:

$$
\left(\begin{array}{c}
e_{11} \\
e_{22} \\
e_{33} \\
-\zeta
\end{array}\right)=\left(\begin{array}{cccc}
S_{11} & S_{12} & S_{13} & -\beta_{1} \\
S_{12} & S_{22} & S_{23} & -\beta_{2} \\
S_{13} & S_{23} & S_{33} & -\beta_{3} \\
-\beta_{1} & -\beta_{2} & -\beta_{3} & \gamma
\end{array}\right)\left(\begin{array}{c}
\sigma_{11} \\
\sigma_{22} \\
\sigma_{33} \\
-p_{f}
\end{array}\right) .
$$

The $e_{i i}$ (no summation over index $i$ ) are principal strains in the $i=1,2,3$ directions. The $\sigma_{i i}$ are the corresponding principal stresses. The pore-fluid pressure is $p_{f}$. The increment of 
fluid content is $\zeta$, and is a dimensionless measure of the number of fluid molecules that move either into or out of the pore space during the deformation process. Its defining equation is:

$$
\zeta \equiv \frac{\delta(\phi V)-\delta V_{f}}{V} \simeq \phi\left(\frac{\delta V_{\phi}}{V_{\phi}}-\frac{\delta V_{f}}{V_{f}}\right),
$$

where $V$ is the overall volume of the fully fluid-saturated porous material at the first moment of consideration, $V_{\phi}=\phi V$ is the pore volume, with $\phi$ being the fluid-saturated porosity within the same volume $V . V_{f}$ is the volume actually occupied by the initial pore-fluid so that, although $V_{f}=V_{\phi}$ initially, these two quantities can differ during the deformation process unless they are constrained (via undrained conditions) to remain the same. The $\delta$ 's are intended to indicate small changes in the quantities immediately following them.

In fact, the undrained state having $\zeta=0$ is a very common condition pertinent for wave propagation studies, especially at ultrasonic frequencies, or for seismic waves at typical exploration frequencies. The drained compliances are $S_{i j}^{d} \equiv S_{i j}$ (consistent with the earlier parts of this paper). Undrained compliances (not yet shown explicitly) will be symbolized by $S_{i j}^{u}$.

An important advantage of the compliance form of the poroelastic equations as shown in (24) is that the only component of this $4 \times 4$ matrix that includes fluid effects is $\gamma$ in the lower right-hand corner. If we invert this matrix to obtain the stiffness version of these equations, the fluid effects get redistributed across all the components because the determinant involved in the matrix inversion process provides a common (fluid-dependent) factor for all the matrix elements in the stiffness form. This is one reason why poroelastic analysis is somewhat simpler in this formulation. 
The drained Reuss average bulk modulus of an anisotropic porous medium is defined by

$$
\frac{1}{K_{R}^{d}}=\sum_{i j=1,2,3} S_{i j}^{d}
$$

To symbolize the Reuss average undrained bulk modulus $K_{R}^{u}$, I have drained compliances replaced by undrained compliances [replace d's by $u$ 's in (26)]. A similar definition of grain Reuss modulus $K_{R}^{g}$, with drained compliances replaced by grain compliances [replace $d$ 's by $g$ 's in (26)] may also be defined, especially when the grains themselves are anisotropic. The alternative Voigt (1928) average [also see Hill (1952)] of the stiffnesses will play no role in this discussion. Off-diagonal coefficients $\beta_{i} \equiv S_{i 1}+S_{i 2}+S_{i 3}-1 / 3 K_{R}^{g}$, where $K_{R}^{g}$ is the Reuss average modulus of the grains (Reuss, 1929). And, finally, the remaining coefficient $\gamma \equiv \sum_{i=1-3} \beta_{i} / B$, where $B$ is the second Skempton (1954) coefficient:

$$
B \equiv \frac{1-K_{R}^{d} / K_{R}^{u}}{1-K_{R}^{d} / K_{R}^{g}}
$$

The shear terms due to twisting motions (i.e., strains $e_{23}, e_{31}, e_{12}$ and stresses $\sigma_{23}$, $\left.\sigma_{31}, \sigma_{12}\right)$ are excluded from this discussion since they typically do not couple to the modes of interest for anisotropic systems having orthotropic symmetry, or any more symmetric system such as transversely isotropic or isotropic. I have also assumed that the true axes of symmetry are known, and make use of them in my current formulation of the problem. Note that the $S_{i j}$ 's are the elements of the compliance matrix $\mathbf{S}$ and are all independent of the fluid, and therefore would be the same if the medium were treated as strictly elastic (i.e., by ignoring the fluid pressure, or assuming that the fluid saturant were air). As mentioned earlier, I typically call these compliances the "drained compliances" and the corresponding matrix the "drained compliance matrix" $\mathbf{S}^{d}$, since then any fluids present do not contribute to the stored mechanical energy if they are free to drain into a surrounding reservoir containing the same type of fluid. In contrast, the undrained compliance matrix 
$\mathbf{S}^{u}$ presupposes that the fluid is trapped (unable to drain from the system into an adjacent reservoir) and therefore contributes in a significant and measurable way to the compliance and stiffness $\left(\mathbf{C}^{u}=\left[\mathbf{S}^{u}\right]^{-1}\right)$, and also therefore to the stored mechanical energy of the undrained system.

Next I find that the off-diagonal $\beta_{i}$ 's must satisfy this sumrule:

$$
\beta_{1}+\beta_{2}+\beta_{3}=\frac{1}{K_{R}^{d}}-\frac{1}{K_{R}^{g}}=\frac{\alpha_{R}}{K_{R}^{d}}
$$

where I have also defined (as for the isotropic case) a Reuss effective stress coefficient (Biot and Willis, 1957):

$$
\alpha_{R} \equiv 1-K_{R}^{d} / K_{R}^{g}
$$

Furthermore, I have the result [consistent with Brown nd Korringa (1975)]

$$
\gamma=\frac{\beta_{1}+\beta_{2}+\beta_{3}}{B}=\frac{\alpha_{R}}{K_{R}^{d}}+\phi\left(\frac{1}{K_{f}}-\frac{1}{K_{R}^{\phi}}\right),
$$

with $\phi$ being porosity. This result follows since I have the rigorous formula in this notation (Berryman, 1997; Berryman and Nakagawa, 2010; Berryman, 2010) showing that Skempton's $B$ coefficient is also given by

$$
B \equiv \frac{\alpha_{R} / K_{R}^{d}}{\alpha_{R} / K_{R}^{d}+\phi\left(1 / K_{f}-1 / K_{R}^{\phi}\right)} .
$$

Note that (27), (30), and (31) all contain dependence on the pore bulk modulus $K_{R}^{\phi}$ that comes into play when the solids present are heterogeneous (Brown and Korringa, 1975) — so the pore response to stress depends in a complicated way on the types and spatial distribution of these different solids, regardless of whether the overall system is isotropic or anisotropic. I should emphasize that all these formulas are rigorous statements based on earlier anisotropic analysis. The appearance of these definitions involving $K_{R}^{d}$ and $\alpha_{R}$ is not an approximation. Rather, this is one useful choice of notation made because it helps to 
clarify the relationships between the rigorous anisotropic formulas and those for isotropic systems.

\section{The $\beta_{i}$ coefficients}

I will now show several useful results for the $\beta_{i}$ coefficients, and then follow the results with a general derivation showing their correctness.

In many important and practical problems, the coefficients $\beta_{i}$ are determined by

$$
\beta_{i}=S_{i 1}^{d}+S_{i 2}^{d}+S_{i 3}^{d}-\frac{1}{3 K_{R}^{g}}
$$

Again, $K_{R}^{g}$ is the Reuss average of the grain modulus, since the local grain modulus is not necessarily assumed uniform - as was mentioned previously. Equation (32) holds true

for homogeneous grains, when $K_{R}^{g}=K^{g}$. But it also holds true for the case when $K_{R}^{g}$ is determined instead by

$$
\frac{1}{K_{R}^{g}} \equiv \sum_{m=1, \ldots, n} \frac{v_{m}}{K_{m}}
$$

where several $(n)$ different types of isotropic grains are assumed to be present, having volume fractions $v_{m}$, respectively, and $\Sigma_{m} v_{m}=1$. However, when the grains themselves are also anisotropic, I need to allow for this possibility in a different way, and this can be accomplished by defining three directional grain bulk moduli determined by:

$$
\frac{1}{3 \bar{K}_{i}^{g}} \equiv S_{i 1}^{g}+S_{i 2}^{g}+S_{i 3}^{g}=S_{1 i}^{g}+S_{2 i}^{g}+S_{3 i}^{g}
$$

for $i=1,2,3$. The second equality follows because the compliance matrix is always symmetric. I call these quantities in (34) the partial grain-compliance sums, and the $\bar{K}_{i}^{g}$ are the directional grain bulk moduli. Then, the formula for (32) is replaced by

$$
\beta_{i}=S_{i 1}^{d}+S_{i 2}^{d}+S_{i 3}^{d}-\frac{1}{3 \bar{K}_{i}^{g}}
$$


Note that the factors of 3 have been correctly accounted for because

$$
\sum_{i=1,2,3} \frac{1}{3 \bar{K}_{i}^{g}}=\frac{1}{K_{R}^{g}}
$$

in agreement with

$$
\frac{1}{K_{R}^{g}}=\sum_{i, j=1,2,3} S_{i j}^{g}
$$

If the three contributions represented by (34) for $i=1,2,3$ happen to be equal, then each equals one-third of the sum in (36).

The preceding results are for perfectly aligned solids grains. If the solids are instead perfectly randomly oriented, then it is an easy exercise to verify that the formulas in (32) hold as before, but now $K_{R}^{g}$ is determined instead by (37), and the three directional grain moduli defined in (34) are equal.

All of these statements about the $\beta_{i}$ 's are easily confirmed by considering applied stresses satisfying $\sigma_{11}=\sigma_{22}=\sigma_{33}=-p_{c}=-p_{f}$, where $p_{c}$ is (external) confining pressure and $p_{f}$ is the (internal) fluid pressure. In this situation, I have from (24), and using (35), that:

$$
-e_{i i}=\left(S_{i 1}^{d}+S_{i 2}^{d}+S_{i 3}^{d}\right) p_{c}+\beta_{i}\left(-p_{f}\right)=\left(S_{i 1}^{g}+S_{i 2}^{g}+S_{i 3}^{g}\right) p_{f} \equiv \frac{p_{f}}{3 \bar{K}_{i}^{g}}
$$

This example is the most general of the three cases discussed, and also holds true for each value of $i=1,2,3$. These are statements about the strain $e_{i i}$ that would be observed in this case, as the formulas must be the same if these anisotropic (or inhomogeneous) grains were immersed in the fluid, while measurements were taken of the strains observed in each of the three directions $i=1,2,3$, during variations of the fluid pressure $p_{f}$. I consider this demonstration to be equivalent to a thought experiment for determining the coefficients, in the same spirit as those originally proposed by Biot and Willis (1957) [also see Stoll (1974)] for the isotropic and homogeneous case. 


\section{Coefficient $\gamma$}

The stated relationship of coefficient $\gamma$ to the other coefficients is easily established since I have already discussed the main issue, which involves determining the role of the various other constants contained in the Skempton (1954) coefficient $B$. This result is

$$
B=\left(\frac{1}{K_{R}^{d}}-\frac{1}{K_{R}^{g}}\right)\left[\left(\frac{1}{K_{R}^{d}}-\frac{1}{K_{R}^{g}}\right)+\phi\left(\frac{1}{K_{f}}-\frac{1}{K_{R}^{\phi}}\right)\right]^{-1} .
$$

Again, from (24), I find that

$$
-\zeta=0=-\left(\beta_{1}+\beta_{2}+\beta_{3}\right) \sigma_{c}-\gamma p_{f},
$$

for undrained boundary conditions. Thus, I again have

$$
\frac{p_{f}}{p_{c}} \equiv B=\frac{\beta_{1}+\beta_{2}+\beta_{3}}{\gamma}
$$

where again $p_{c}=-\sigma_{c}$ is the confining pressure. Therefore, the scalar coefficient $\gamma$ is determined immediately and given explicitly by

$$
\gamma=\frac{\beta_{1}+\beta_{2}+\beta_{3}}{B}=\frac{\alpha_{R} / K_{R}^{d}}{B}=\alpha_{R} / K_{R}^{d}+\phi\left(\frac{1}{K_{f}}-\frac{1}{K_{R}^{\phi}}\right) .
$$

Alternatively, I can consider the identity

$$
B=\frac{\alpha_{R}}{\gamma K_{R}^{d}}
$$

for the Skempton (1954) B coefficient that also follows easily from the same derivation.

I have now determined the physical significance of all the coefficients in the poroelastic matrix (24). These results are as general as possible without considering poroelastic anisotropies that have less than orthotropic symmetry, while also taking advantage of the assumption that I do often know (or can deduce from measurements) the three directions of the principal axes of symmetry. 


\section{Inverting poroelastic compliance}

The matrix in (24) is in compliance form and has extremely simple poroelastic behavior in the sense that all the fluid-mechanical effects appear only in the single coefficient $\gamma$. I can simplify the notation a little more by lumping some coefficients together, combining the $3 \times 3$ submatrix in the upper left corner of the matrix in $(24)$ as $\mathbf{S}$, and defining the column vector $\mathbf{b}$ by

$$
\mathbf{b}^{T} \equiv\left(\beta_{1}, \beta_{2}, \beta_{3}\right)
$$

The resulting $4 \times 4$ matrix and its inverse are now related by:

$$
\left(\begin{array}{cc}
\mathbf{S} & -\mathbf{b} \\
-\mathbf{b}^{T} & \gamma
\end{array}\right)=\left(\begin{array}{ll}
\mathbf{A} & \mathbf{q} \\
\mathbf{q}^{T} & z
\end{array}\right)^{-1},
$$

where the elements of the inverse matrix can be shown to be written in terms of drained stiff-

ness matrix $\mathbf{C}^{d}=\mathbf{C}=\mathbf{S}^{-1}$ by introducing three components: $(a)$ scalar $z=\left[\gamma-\mathbf{b}^{T} \mathbf{C b}\right]^{-1}$, (b) column vector $\mathbf{q}=z \mathbf{C b}$, and $(c)$ undrained $3 \times 3$ stiffness matrix (i.e., the pertinent one connecting the principal strains to principal stresses)

$$
\mathbf{A}=\mathbf{C}+z \mathbf{C b b}^{T} \mathbf{C}=\mathbf{C}^{d}+z^{-1} \mathbf{q q}^{T} \equiv \mathbf{C}^{u}
$$

[While these results are all easy to verify once known, the reader will find they are harder to show directly from the general form of (45).] The drained stiffness is $\mathbf{C}^{d}=\mathbf{C}$ by definition, and $\mathbf{A}=\mathbf{C}^{u}$ is clearly undrained stiffness by construction. This fact comes from the undrained identity

$$
\left(\begin{array}{cc}
\mathbf{A} & \mathbf{q} \\
\mathbf{q}^{T} & z
\end{array}\right)\left(\begin{array}{c}
\mathbf{E} \\
0
\end{array}\right)=\left(\begin{array}{c}
\boldsymbol{\Sigma} \\
-p_{f}
\end{array}\right),
$$

which follows from (24) and (45) with the definitions:

$$
\mathbf{E}^{T}=\left(e_{11}, e_{22}, e_{33}\right) \text { and } \quad \boldsymbol{\Sigma}^{T}=\left(\sigma_{11}, \sigma_{22}, \sigma_{33}\right) .
$$


These results are the same as those of Gassmann (1951a,b) for anisotropic porous media, although his results were presented in a different form, which is fundamentally the same as (46).

Equation (45) also shows that the decoupling of the fluid effects occurs only in the compliance form (24) of the equations, and never in the stiffness (inverse) form for the poroelasticity equations. Fluid factors appear everywhere in the stiffness matrix form because the inverse relation requires division by the determinant of the $4 \times 4$ in compliance form. This determinant (a scalar) depends explicitly on the fluid bulk modulus, and therefore spreads the fluid effects across the entire $4 \times 4$ stiffness matrix.

From these results, it easily follows that

$$
\mathbf{S}^{d}=\mathbf{S}^{u}+\gamma^{-1} \mathbf{b b}^{T}
$$

This formula emphasizes the fact that the drained compliance matrix can be found directly from knowledge of the inverse of undrained compliance, and the still unknown, but sometimes relatively easy to estimate, values of $\gamma$, together with the three distinct off-diagonal orthotropic $\beta_{i}$ coefficients, for $i=1,2,3$.

\section{Orthotropic example with homogeneous grains: Deducing coefficients from measurements}

Now, further progress is made by considering the Reuss average again for both of the orthotropic drained and undrained compliances:

$$
\frac{1}{K_{R}^{d}} \equiv \sum_{i, j=1,2,3} S_{i j}^{d},
$$


and

$$
\frac{1}{K_{R}^{u}} \equiv \sum_{i, j=1,2,3} S_{i j}^{u}
$$

These effective moduli are the Reuss averages of the nine compliances in the upper left $3 \times 3$ of the full (including the uncoupled shear components) $6 \times 6$ compliance matrix for the two cases, respectively, when the pore fluid is allowed to drain from the porous system, and when the pore fluid is trapped by a jacketing material, and so undrained.

Although the significance of the formula in the orthotropic case is somewhat different now, I find again that

$$
\beta_{1}+\beta_{2}+\beta_{3}=\frac{1}{K_{R}^{d}}-\frac{1}{K_{R}^{g}}=\frac{\alpha_{R}}{K_{R}^{d}},
$$

if I also define a Reuss effective stress coefficient:

$$
\alpha_{R} \equiv 1-K_{R}^{d} / K_{R}^{g}
$$

by analogy to the isotropic case. Furthermore, I have

$$
\gamma=\frac{\beta_{1}+\beta_{2}+\beta_{3}}{B}=\frac{\alpha_{R}}{K_{R}^{d}}+\phi\left(\frac{1}{K_{f}}-\frac{1}{K_{R}^{g}}\right),
$$

since I have the rigorous result in this notation (Berryman, 1997) that Skempton's $B$ coefficient (Skempton, 1954) is given by

$$
B \equiv \frac{1-K_{R}^{d} / K_{R}^{u}}{1-K_{R}^{d} / K_{R}^{g}}=\frac{\alpha_{R} / K_{R}^{d}}{\alpha_{R} / K_{R}^{d}+\phi\left(1 / K_{f}-1 / K_{R}^{g}\right)}
$$

Note that, from (52) and (54), it follows that $\gamma^{-1}=\frac{B K_{R}^{d}}{\alpha_{R}}$ [also see (43)]. So I can now rewrite (49) to give the formal relationship

$$
S_{i j}^{d}=S_{i j}^{u}+\frac{B K_{R}^{d}}{\alpha_{R}} \beta_{i} \beta_{j}, \quad \text { for } \quad i, j=1,2,3 .
$$


At this point in the analysis, I know everything needed except for the three coefficients $\beta_{i}$, for $i=1,2,3$. But, by using appropriate sums of (56), I find that

$$
\beta_{i}=S_{i 1}^{d}+S_{i 2}^{d}+S_{i 3}^{d}-\frac{1}{3 K_{R}^{g}}=S_{i 1}^{u}+S_{i 2}^{u}+S_{i 3}^{u}-\frac{1}{3 K_{R}^{g}}+B \beta_{i} .
$$

Rearranging (57), I find that

$$
\beta_{i}(1-B)=S_{i 1}^{u}+S_{i 2}^{u}+S_{i 3}^{u}-\frac{1}{3 K_{R}^{g}}
$$

Formula (55) for the Skempton (1954) coefficient determines $B$ exactly in terms of (presumed) known quantities. In the present example, the Skempton coefficient $B$, however, was not assumed to be known, since for homogeneous grains I can compute $K_{R}^{d}$ relatively easily, and then $B$ follows since I also know $K_{R}^{g}$. For the case of heterogeneous or anisotropic

grains, the necessary introduction of the additional pore modulus $K_{R}^{\phi}$ requires still more measured data, and it turns out that the next easiest quantity to measure is $B$ itself as can easily happen in the isotropic case. So, all three $\beta_{i}$ 's (which are themselves drained constants) and $\gamma$ are now precisely determined. All the remaining drained compliances $S_{i j}^{d}$ can then be found directly from (56).

\section{Homogeneous grains and pores: Deducing anisotropic drained constants from undrained}

I am now ready to complete the development of an analogy between the isotropic and anisotropic Gassmann (1951) equations for the case of homogeneous grains. In particular, the equation for the suspension modulus in

$$
K_{\text {susp }}=\left[\frac{1-\phi}{K^{g}}+\frac{\phi}{K_{f}}\right]^{-1}
$$


does not change at all. In contrast, the equation for the effective undrained bulk modulus $K^{u}$, as shown in two versions of the same formula

$$
K^{u}=K^{d}+\alpha^{2} /\left[(\alpha-\phi) / K^{g}+\phi / K_{f}\right]
$$

and

$$
K^{u}=K^{d}+\frac{\left(1-K^{d} / K^{g}\right)^{2}}{1 / K_{\text {susp }}-K^{d} /\left(K^{g}\right)^{2}},
$$

is altered only because the relationship is now between the Reuss averages $K_{R}^{u}$ and $K_{R}^{d}$ of these quantities. This result is completely analogous to (61), and so will not be shown here.

The remainder of the argument is virtually identical to the isotropic case, so I obtain:

$$
K_{R}^{d}=\left(\frac{K_{R}^{u}}{K_{\text {susp }}}-1\right)\left[1 / K_{\text {susp }}-2 / K_{R}^{g}+K_{R}^{u} /\left(K_{R}^{g}\right)^{2}\right]^{-1} .
$$

This formula shows how to invert for drained Reuss bulk modulus $K_{R}^{d}$ from knowledge of $K_{R}^{u}, \phi, K_{f}$ and $K_{R}^{g}$ in an anisotropic (up to orthotropic) poroelastic system.

Clearly, this formula does not yet provide the individual compliance matrix elements $S_{i j}^{d}$ directly. Nevertheless, the rest of the steps follow easily once this rigorous result is known.

To finish the analysis, I make use of the newly computed value of $K_{R}^{d}$, and substitute this value into the formula for $B$, which in this case is:

$$
B=\frac{1-K_{R}^{d} / K_{R}^{u}}{1-K_{R}^{d} / K_{R}^{g}}
$$

This formula is the same as the results found previously (but written differently) in (27), (31), (39), (43), and (55). Once I know Skempton coefficient $B$, this scalar can be substituted into (58) in order to determine the $\beta_{i}$ coefficients for $i=1,2,3$. The remaining coefficient is $\gamma=\alpha_{R} / B K_{R}^{d}$. So I have shown that the critical step in this procedure was determining the value of the drained Reuss bulk modulus $K_{R}^{d}$. 


\section{Heterogeneous grains and pores: Deducing anisotropic drained constants from undrained}

One difficulty for heterogeneous grains comes from the additional constant $K_{R}^{\phi}$ which I do not immediately know how to determine independently from the other poroelastic measurements. But this fundamental problem is actually no different for the anisotropic case than it was for the isotropic one, and the solution is also the same. In both cases, I need more information, and in both cases the necessary information will most likely come from our knowledge (through new measurements, or perhaps via tabulated values for various rock types) of the Skempton (1954) coefficient $B$. If I assume that $B$ can be directly measured (which is plausible for laboratory measurements, since $B=p_{f} / p_{c}$ in the undrained case when a uniform confining pressure is applied to the system), then the problem is completely solved, because $B$ is the key to solving for the coefficients $\beta_{i}$ in (58). The only new difficulty is that the terms of the form $1 / 3 K_{R}^{g}$ must also be replaced by the partial grain compliance sums $\frac{1}{3 \bar{K}_{i}^{g}}$, as shown in (35). So I now have

$$
\beta_{i}=S_{i 1}^{d}+S_{i 2}^{d}+S_{i 3}^{d}-\frac{1}{3 \bar{K}_{i}^{g}}=S_{i 1}^{u}+S_{i 2}^{u}+S_{i 3}^{u}-\frac{1}{3 \bar{K}_{i}^{g}}+B \beta_{i} .
$$

Rearranging, I find that, for heterogeneous grains, the result is:

$$
\beta_{i}(1-B)=S_{i 1}^{u}+S_{i 2}^{u}+S_{i 3}^{u}-\frac{1}{3 \bar{K}_{i}^{g}}
$$

So, I am almost done now, but I still need either to determine the values of the anisotropic grain correction terms $\frac{1}{3 \bar{K}_{i}^{g}}$, or to find some way of avoiding the necessity of doing so.

In principle, this goal can be attained experimentally in laboratory tests by actually performing measurements on the porous sample while applying the same pressure outside and inside the porous sample. Then, measurements of the change in strain in the three 
orthogonal directions $i=1,2,3$ would provide direct measures of the quantities $\bar{K}_{i}^{g}$ needed. So this approach is one that is entirely feasible.

An alternative would be to perform shear tests by applying nonzero deviatoric stress changes (Skempton, 1954; Lockner and Stanchits, 2002). The undrained fluid pressure is given by $p_{f}=B p_{c}=B\left(-\sigma_{m}\right)$, where the mean stress is $\sigma_{m}=\left(\sigma_{11}+\sigma_{22}+\sigma_{33}\right) / 3$. But, if the $\sigma_{i i}$ 's are not uniform, then there are also deviatoric stresses present, due to the nonuniformity of the principal stresses.

\section{Triaxial testing geometry for laboratory measurements}

One common example of this type of measurement uses triaxial testing (Lockner and Stanchits, 2002), where a two-sided confining stress is defined as $\sigma_{22}=\sigma_{33}$, and then the deviatoric stress is determined by

$$
\sigma_{\text {dev }} \equiv\left(\sigma_{11}-\sigma_{33}\right) / 2
$$

In this situation, the general equation relating undrained pressure to the confining stresses is given by:

$$
-p_{f}=B \sigma_{m}+2\left(A-\frac{1}{3}\right) B \sigma_{d e v}
$$

where the only new symbol is the first coefficient $A$ of Skempton (1954).

It is not difficult to show that, in terms of our previous definitions for the triaxial testing geometry, the coefficient $A$ is given precisely by the ratio

$$
A \equiv \frac{\beta_{1}}{\beta_{1}+\beta_{2}+\beta_{3}} .
$$

For an isotropic system, $A=1 / 3$, so this contribution always vanishes in (67). This fact explains why this coefficient does not appear for isotropic analysis of poroelastic systems. 
Note that there is no assumption here that the poroelastic system itself is necessarily transversely isotropic. Only the prescribed equality (in a laboratory setting) of the two applied transverse stresses, $\sigma_{22}$ and $\sigma_{33}$, is assumed. Then, the formula (67) follows directly from the equations already presented.

\section{Measurements of Skempton's $B$ coefficient}

There are many good sources of information about measured values of Skempton's $B$ coefficient. I will not attempt to review these here. One well-known reference that contains some values and discussion is Rice and Cleary (1976). The text by Wang (2000) contains tables of values taken from many sources, some of which are quoted here in TABLE 4 . One series of related contributions on the same subject includes papers by Green and Wang (1986), Berge et al. (1993), Hart and Wang (1995, 2001). A very useful Ph. D. thesis with an unpublished chapter on this topic was contributed by Hornby (1995). Although it might appear from this listing that data are plentiful, it is clear from a quick scan of these papers, books, and theses that more data of these types are certainly needed, especially for rocks of particular interest in the seismic exploration context.

\section{CONCLUSIONS}

In this paper I have treated various methods for quantifying the geomechanical effects of fracture sets on reservoirs. Special emphasis has been given to recent work, and also to the influence that work of Michael Schoenberg has had on this subject. I conclude that these methods provide a consistent and accurate picture of the influence of fractures on wave propagation in many cases, and that the various methods in use, although sometimes 
presented quite differently, are very closely related both conceptually and also in terms of the quantitative predictions. Generalization to fractures filled with fluids can also be treated rather easily by incorporating the well-known Skempton's $A$ and $B$ coefficients in order to account rigorously for the reduced weakening effect (because of the stiffening effect of the fluid saturant) of fractures when they are liquid-filled.

Of course, elastic orthotropy is not universal in the earth, so it is not appropriate to assume that all the problems in exploration seismology will be solved using such relatively simple models. One final conclusion must be that, although good progress has been made, there is also clearly more work to be done relative to the influence of fluids and fractures on seismic waves in more general anisotropic media than the ones considered specifically here.

\section{ACKNOWLEDGMENTS}

I thank Atilla Aydin, Vladimir Grechka, Mark Kachanov, Francis Muir, Seiji Nakagawa, Steven R. Pride, Colin Sayers, Leon Thomsen, Ilya Tsvankin, and especially Michael Schoenberg - to whose memory this paper is dedicated — for many helpful, stimulating, and enlightening discussions about fractures and anisotropy. Work performed under the auspices of the U.S. Department of Energy, at the Lawrence Berkeley National Laboratory under Contract No. DE-AC02-05CH11231. Support was provided specifically by the Geosciences Research Program of the DOE Office of Basic Energy Sciences, Division of Chemical Sciences, Geosciences and Biosciences.

\section{REFERENCES}

Backus, G. E., 1962, Long-wave elastic anisotropy produced by horizontal layering: Journal of Geophysical Research, 67, no. 11, 4427-4440. 
Bakulin, A., V. Grechka, and I. Tsvankin, 2000, Estimation of fracture parameters from reflection seismic data - Part I: HTI model due to a single fracture set: Geophysics, 65, no. $6,1788-1802$.

Bažant, Z. P. and J. Planas, 1998, Fracture and Size Effect in Concrete and Other Quasibrittle Materials: CRC Press, Boca Raton, Florida.

Berge, P. A., H. F. Wang, and B. P. Bonner, 1993, Laboratory measurements of a complete set of poroelastic moduli for Berea sandstone and Indiana limestone: International Journal of Rock Mechanics, Mining Sciences, and Geomechanics Abstracts, 30, no. 7, 1135-1141.

Berryman, J. G., 1979, Long-wave elastic anisotropy in transversely isotropic media: Geophysics, 44, no. 5, 896-917.

1997, Transversely isotropic elasticity and poroelasticity arising from thin isotropic layers, in Teng, Y.-C., E.-C. Shang, Y.-H. Pao, M. H. Schultz, and A. D. Pierce, eds., Theoretical and Computational Acoustics 1997, 457-474, World Scientific, Newark, NJ.

2005, Bounds and self-consistent estimates for elastic constants of random polycrystals with hexagonal, trigonal, and tetragonal symmetries: Journal of the Mechanics and Physics of Solids, 53, no. 10, 2121-2173.

2007, Seismic waves in rocks with fluids and fractures: Geophysical Journal International, 171, 954-974.

2008, Exact seismic velocities for transversely isotropic media and extended Thomsen formulas for stronger anisotropies: Geophysics, 73, no. 1, D1-D10.

2010, Poroelastic measurement schemes resulting in complete data sets for granular and other anisotropic porous media: International Journal of Engineering Science, 48, 
no. 4, 446-459.

Berryman, J .G. and A. Aydin, 2010, Quasi-static analysis of elastic behavior for some systems having higher fracture densities: International Journal for Numerical and Analytical Methods in Geomechanics. (DOI: 10.1002/nag.874).

Berryman, J. G. and V. Grechka, 2006, Random polycrystals of grains containing cracks: Model of quasistatic elastic behavior for fractured systems: Journal of Applied Physics, 100, no. 11, 113527.

Berryman, J. G. and S. Nakagawa, 2010, Inverse problem in anisotropic poroelasticity: Drained constants from undrained ultrasound measurements: Journal of the Acoustical Society of America, 127, no. 2, 720-729.

Biot, M. A. and D. G. Willis, 1957, The elastic coefficients of the theory of consolidation: ASME Journal of Applied Mechanics, 24, 594-601.

Brillouin, L., 1946, Wave Propagation in Periodic Structures: Dover, New York.

Bristow, J. 1960, Microcracks, and the static and dynamic elastic constants of annealed and heavily cold-worked metals: British Journal of Applied Physics, 11, no. 2, 81-85.

Brown, R. J. S. and J. Korringa, 1975, On the dependence of the elastic properties of a porous rock on the compressibility of the pore fluid: Geophysics, 40, no. 4, 608-616.

Budiansky, B. and R. J. O'Connell, 1976, Elastic moduli of a cracked solid: International Journal of Solids and Structures, 12, no. 2, 81-97.

Cheng, A. H.-D., 1997, Material coefficients of anisotropic poroelasticity: International Journal of Rock Mechanics and Mining Sciences, 34, no. 2, 199-205.

Daley, T. M., M. Schoenberg, J. Rutqvist, and K. T. Nihei, 2006, Fractured reservoirs: An 
analysis of coupled elastodynamic and permeability changes from pore-pressure variation: Geophysics, 71, no. 5, O33-O41.

Eshelby, J. D., 1957, The determination of the elastic field of an ellipsoidal inclusion, and related problems: Proceedings of the Royal Society of London A, 241, no. 1226, 376-396. Gassmann, F., 1951a, On elasticity of porous media: in Pelissier, M. A., H. Hoeber, N. van de Coevering, and I. F. Jones, eds., Classics of Elastic Wave Theory, volume 24, 389-407, Society of Exploration Geophysicists, Tulsa, OK.

, 1951b, Über die Elastizitat poröser Medien: Vierteljahrsschrift der Naturforschenden Gesellschaft in Zürich, 96, 1-23.

1964, Introduction to seismic travel-time methods in anisotropic media: Pure and Applied Geophysics, 58, 53-112.

Grechka, V., I. Vasconcelos, and M. Kachanov, 2006, Influence of crack shape on the effective elasticity of fractured rocks: Geophysics, 71, no. 5, D153-D160.

Green, D. H. and H. F. Wang, 1986, Fluid pressure response to undrained compression in saturated sedimentary rock: Geophysics, 51, no. 4, 948-956.

Hart, D. J. and H. F. Wang, 1995, Laboratory measurements of a complete set of poroelastic moduli for Berea sandstone and Indiana limestone: Journal of Geophysical Research, 100, no. B9, 17741-17751.

2001, A single test method for determination of poroelastic constants and flow parameters in rock with low hydraulic conductivities: International Journal of Rock Mechanics and Mining Sciences, 38, no. 4, 577-583.

Hill, R., 1952, The elastic behaviour of crystalline aggregate: Proceedings of the Physical 
Society of London, A65, 349-354.

Hornby, B. E., 1995, The Elastic Properties of Shales: Ph.D., Cambridge University, Cambridge, England (Chapter 8).

Kachanov, M., 1980, Continuum model of medium with cracks: Journal of the Engineering Mechanics Division of ASCE, 106, no. 5, 1039-1051.

Lockner, D. A. and S. A. Stanchits, 2002, Undrained poroelastic response of sandstones to deviatoric stress change: Journal of Geophysical Research, 107, no. B12, 2353-1-2353-14.

Reuss, A., 1929, Berechung der Fliessgrenze von Mischkristallen: Z. Angew. Math. Mech., 9,55 .

Rice, J. R. and M. P. Cleary, 1976, Some basic stress-diffusion solutions for fluid-saturated elastic porous media with compressible constituents: Reviews of Geophysics and Space Physics, 14, no. 2, 227-241.

Rüger, A., 2002, Reflection Coefficients and Azimuthal AVO Analysis in Anisotropic Media: Society of Exploration Geophysicists, Tulsa, OK.

Sayers, C. M. and M. Kachanov, 1991, A simple technique for finding effective elastic constants of cracked solids for arbitrary crack orientation statistics, International Journal of Solids and Structures, 27, no. 6, 671-680.

1995, Microcrack-induced elastic wave anisotropy of brittle rocks: Journal of Geophysical Research, 100, no. B3, 4149-4156.

Sayers, C. M., A. D. Taleghani, and J. Adachi, 2009, The effect of mineralization on the ratio of normal to tangential compliance of fractures: Geophysical Prospecting, 57, no. 3, $439-446$. 
Schoenberg, M., 1980, Elastic wave behavior across linear slip interfaces: Journal of the Acoustical Society of America, 68, no. 5, 1516-1521.

Schoenberg, M. and K. Helbig, 1997, Orthorhombic media: Modeling elastic wave behavior in a vertically fractured earth: Geophysics, 62, no. 6, 1954-1974.

Schoenberg, M. and F. Muir, 1989, A calculus for finely layered anisotropic media: Geophysics, 54, no. 4, 581-589.

Schoenberg, M., and C. M. Sayers, 1995, Seismic anisotropy of fractured rock: Geophysics, 60, no. 1, 204-211.

Skempton, A. W., 1954, The pore-pressure coefficients $A$ and B: Geotechnique, 4, 143-147.

Stoll, R. D., 1974, Acoustic waves in saturated sediments, in Physics of Sound in Marine Sediments, 19-39, Plenum, New York.

Thomsen, L., 1986, Weak elastic anisotropy: Geophysics, 51, no. 10, 1954-1966. 2002, Understanding Seismic Anisotropy in Exploration and Exploitataion: Society of Exploration Geophysicists, Tulsa, OK.

Tsvankin, I., 1997, Anisotropic parameters and $P$-wave velocity for orthorhombic media: Geophysics, 62, no. 4, 1292-1309. 2005, Seismic Signatures and Analysis of Reflection Data in Anisotropic Media: Elsevier, Oxford, UK.

Voigt, W., 1928, Lehrbuch der Kristallphysik: Teubner, Leipzig.

Wang, H. F., 2000, Theory of Linear Poroelasticity with Applications to Geomechanics and Hydrogeology: Princeton University Press, Princeton, NJ. 
Wood, A. W., 1948, A Textbook of Sound: Bell, London, p. 360.

Zhu, X. and G. A. McMechan, 1990, Direct estimation of the bulk modulus of the frame in a fluid-saturated elastic medium by Biot theory: 60th International Meeting, Expanded Abstracts, Society of Exploration Geophysicists, Tulsa, OK, 787-790. 


\section{APPENDIX A - POROELASTICITY WITH HOMOGENEOUS SOLID BACKGROUND}

Gassmann's famous poroelasticity equation (Gassmann, 1951a,b) relating drained bulk modulus $K^{d}$ to undrained modulus $K^{u}$ for homogeneous isotropic systems is sometimes written in the form

$$
K^{u}=K^{d}+\alpha^{2} /\left[(\alpha-\phi) / K^{g}+\phi / K_{f}\right],
$$

where $\alpha \equiv 1-K^{d} / K^{g}$ is the effective stress coefficient or Biot-Willis coefficient (Biot and Willis, 1957), $K^{g}$ is the solid modulus of the grains (initially assumed homogeneous), $K_{f}$ is the pore-fluid modulus, and $\phi$ is the porosity. The formula becomes more complicated if the solids constituting the porous medium are heterogeneous (Brown and Korringa, 1975) see Appendix B for a discussion. This important generalization is also treated in the main text. I present only the case for homogeneous solids in this Appendix, together with some references to the results for heterogeneous media.

For notational convenience, I introduce a modulus for a fluid suspension having the same solid and fluid components as well as the same porosity as that in the Gassmann result, but having drained modulus $K^{d} \equiv 0$. Then I find that the effective modulus is given by

$$
K_{\text {susp }}=\left[\frac{1-\phi}{K^{g}}+\frac{\phi}{K_{f}}\right]^{-1} .
$$

In the homogeneous case, this fact follows directly from Gassmann's formula (69) by setting $K^{d}=0$ everywhere, since then $K^{u}=K_{\text {susp }}$. But of course this result is also well-known in mechanics and acoustics (Wood, 1948) for these types of fluid-solid suspensions.

Rewriting Gassmann's formula in these terms, I find

$$
K^{u}=K^{d}+\frac{\left(1-K^{d} / K^{g}\right)^{2}}{1 / K_{\text {susp }}-K^{d} /\left(K^{g}\right)^{2}} .
$$


All explicit porosity $\phi$ dependence is now imbedded in the modulus $K_{\text {susp }}$. From this form of the undrained equation, it is not hard to invert for the drained modulus and thereby show that it is expressible as:

$$
K^{d}=\left(\frac{K^{u}}{K_{\text {susp }}}-1\right)\left[1 / K_{\text {susp }}-2 / K^{g}+K^{u} /\left(K^{g}\right)^{2}\right] \cdot \cdot^{-1}
$$

The transition from (71) to (72) involved only the solution of an equation linear in $K^{d}$. Thus, the drained modulus in homogeneous media can be deduced from measurements of the undrained modulus, together with knowledge of $\phi, K_{f}$, and $K^{g}$. This result was apparently first derived by Zhu and McMechan (1990), but published only in a conference proceedings.

Although result (72) just presented applies only to isotropic and homogeneous systems, there are similar results (as is shown in the main text) for anisotropic systems (up to orthotropic symmetry), and also for heterogeneous systems. Furthermore, the orthotropic poroelastic system of equations can additionally be inverted for all the drained constants. Demonstrating these facts for homogeneous porous media is the focus of this Appendix, with special emphasis being given to fractured systems.

If the overall porous medium is anisotropic either due to some preferential alignment of the constituent anisotropic particles or due to externally imposed stress (such as a gravity field and weight of overburden, for example), I reconsider the orthorhombic anisotropic version of the poroelastic equations presented in (24). The $e_{i i}$ (no summation over repeated indices) are principal strains in the $i=1,2,3$ directions. The $\sigma_{i i}$ (again no summation over repeated indices) are the corresponding principal stresses. The fluid pressure is $p_{f}$. The increment of fluid content is $\zeta$, and is defined in (25). The drained compliances are 
$S_{i j}^{d} \equiv S_{i j}$. The drained Reuss average bulk modulus (Reuss, 1929) is also defined by

$$
\frac{1}{K_{R}^{d}}=\sum_{i j=1,2,3} S_{i j}^{d}
$$

Undrained compliances will be symbolized by $S_{i j}^{u}$. For the Reuss average undrained bulk modulus $K_{R}^{u}$, I have drained compliances replaced by undrained compliances in a formula otherwise identical in form to (73), with symbol $d$ replaced by $u$. Off-diagonal coefficients $\beta_{i}=S_{i 1}+S_{i 2}+S_{i 3}-1 / 3 K_{R}^{g}$, where $K_{R}^{g}$ is again the Reuss average modulus of the grains - simply replace $d$ 's with $g$ 's in (73) to get $K_{R}^{g}$. The alternative (Voigt, 1928) average [also see Hill (1952)] of the stiffnesses does not appear. And, finally, coefficient $\gamma=\sum_{i=1-3} \beta_{i} / B$ in (24), where $B$ is the second Skempton coefficient (Skempton, 1954).

The shear terms due to twisting motions (i.e., strains $e_{23}, e_{31}, e_{12}$ and stresses $\sigma_{23}, \sigma_{31}$, $\left.\sigma_{12}\right)$ are excluded from the present discussion because they typically do not couple to the modes of interest for anisotropic systems having orthotropic symmetry, or more symmetric systems such as those having transversely isotropic or isotropic symmetry.

Summing the off-diagonal coefficients $\beta_{i}$, I now have

$$
\beta_{1}+\beta_{2}+\beta_{3}=\frac{1}{K_{R}^{d}}-\frac{1}{K_{R}^{g}}=\frac{\alpha_{R}}{K_{R}^{d}}
$$

I also define (as in the isotropic case) a Reuss effective stress coefficient: $\alpha_{R} \equiv 1-K_{R}^{d} / K_{R}^{g}$. Furthermore, I have

$$
\gamma=\frac{\beta_{1}+\beta_{2}+\beta_{3}}{B}=\frac{\alpha_{R}}{K_{R}^{d}}+\phi\left(\frac{1}{K_{f}}-\frac{1}{K_{R}^{g}}\right),
$$

since a rigorous result in this notation (Berryman, 1997) is that Skempton's $B$ coefficient satisfies:

$$
B \equiv \frac{1-K_{R}^{d} / K_{R}^{u}}{1-K_{R}^{d} / K_{R}^{g}}=\frac{\alpha_{R} / K_{R}^{d}}{\alpha_{R} / K_{R}^{d}+\phi\left(1 / K_{f}-1 / K_{R}^{g}\right)}
$$


More general versions (Brown and Korringa, 1975) of the $B$ definition include another bulk modulus $K_{R}^{\phi}$ for pore response that differs from the grain response if the medium consists of a heterogeneous collection of grains, but this difference is beyond the scope of this Appendix (but see Appendix B). With the exception of this caveat, all these formulas presented are rigorous statements based on anisotropic analysis. The appearances of the Reuss-based expressions $K_{R}^{d}$ and $\alpha_{R}$ are not approximations.

I next provide several results for the off-diagonal $\beta_{i}$ coefficients, and then follow the results with a demonstration of their validity.

The coefficients $\beta_{i}$ are often determined by

$$
\beta_{i}=S_{i 1}^{d}+S_{i 2}^{d}+S_{i 3}^{d}-\frac{1}{3 K_{R}^{g}} .
$$

Again, $K_{R}^{g}$ is the Reuss average of the grain modulus. Equation (77) holds true for homogeneous grains, such that $K_{R}^{g}=K^{g}$. However, when the grains themselves are anisotropic, I also need to allow for this possibility by defining three directional grain bulk moduli determined by:

$$
\frac{1}{3 \bar{K}_{i}^{g}} \equiv S_{i 1}^{g}+S_{i 2}^{g}+S_{i 3}^{g}=S_{1 i}^{g}+S_{2 i}^{g}+S_{3 i}^{g}
$$

for $i=1,2,3$. The second equality follows because the compliance matrix is always symmetric. I call these quantities in (78) the partial grain-compliance sums, and the $\bar{K}_{i}^{g}$ are pertinent directional grain bulk moduli. Then, the formula for (77) is replaced by

$$
\beta_{i}=S_{i 1}^{d}+S_{i 2}^{d}+S_{i 3}^{d}-\frac{1}{3 \bar{K}_{i}^{g}}
$$

The preceding results are for perfectly aligned grains. If the grains are instead perfectly randomly oriented, then it is clear that the formulas in (77) hold as before, but now the 
definition of $K_{R}^{g}$ must be reformulated as

$$
\frac{1}{K_{R}^{g}}=\sum_{i j=1,2,3} S_{i j}^{g} .
$$

If the actual heterogeneous, anisotropic rock material has internal organization that is neither perfectly aligned, nor perfectly random, then an effective medium theory needs to be used to estimate these effective grain moduli (Berryman, 2005). I will not pursue this specialized thread of the analysis any further here.

All of these statements about the $\beta_{i}$ are easily proven by considering the situation when $\sigma_{11}=\sigma_{22}=\sigma_{33}=-p_{c}=-p_{f}$. Because then, from (24), I have:

$$
\begin{aligned}
-e_{i i} & =\left(S_{i 1}^{d}+S_{i 2}^{d}+S_{i 3}^{d}\right) p_{c}+\beta_{i}\left(-p_{f}\right) \\
& =\left(S_{i 1}^{g}+S_{i 2}^{g}+S_{i 3}^{g}\right) p_{f} \equiv \frac{p_{f}}{3 \bar{K}_{i}^{g}},
\end{aligned}
$$

in the most general of the three cases discussed, and holding true for each value of $i=$ $1,2,3$. This result is a statement about the $i$-th diagonal component of strain $e_{i i}$ that would be observed in such circumstances, as it must be the same if these anisotropic (or inhomogeneous) grains were immersed in the fluid, while measurements were taken of the strains observed in each of the three directions $i=1,2,3$, during variations of the fluid pressure $p_{f}$. This "proof" is a thought experiment for determining the coefficients, in the same spirit as the thought experiments proposed originally by Biot and Willis (1957) for the isotropic and homogeneous case.

The relationship of coefficient $\gamma$ to the other coefficients is easily established because I have already discussed the main issue, which involves determining the role of the various other constants contained in Skempton's coefficient B (Skempton, 1954). Again, from (24), I find that

$$
-\zeta=0=-\left(\beta_{1}+\beta_{2}+\beta_{3}\right) \sigma_{c}-\gamma p_{f}
$$


for undrained boundary conditions. Thus, I find again that

$$
\frac{p_{f}}{p_{c}} \equiv B=\frac{\beta_{1}+\beta_{2}+\beta_{3}}{\gamma},
$$

where $p_{c}=-\sigma_{c}$ is the confining pressure. Then, the scalar coefficient $\gamma$ is determined immediately, and is given by

$$
\gamma=\frac{\beta_{1}+\beta_{2}+\beta_{3}}{B}=\frac{\alpha_{R} / K_{R}^{d}}{B}=\alpha_{R} / K_{R}^{d}+\phi\left(\frac{1}{K_{f}}-\frac{1}{K_{R}^{g}}\right) .
$$

Alternatively, I might say that Skempton's coefficient

$$
B=\frac{\alpha_{R}}{\gamma K_{R}^{d}}
$$

I have now determined the physical/mechanical significance of all the coefficients in the poroelastic matrix (24) in terms of measurable quantities. These results are as general as possible without considering poroelastic anisotropies that have less than orthotropic symmetry and without allowing for either heterogeneous grains or special sets of grain orientations. I have also taken advantage of the assumption that the three directions of the principal axes of symmetry are typically known in order to simplify the presentation.

Now the issue becomes whether or not it is possible to deduce all the drained constants from measured undrained ones. This question takes the form of a type of poroelastic inverse problem. From (24) and previous definitions, the drained compliances satisfy:

$$
S_{i j}^{d}=S_{i j}^{u}+\frac{B K_{R}^{d}}{\alpha_{R}} \beta_{i} \beta_{j}
$$

The only unknown component on the right hand side of (86) is the undrained constants, but I assume these have actually been measured by doing undrained wave propagation experiments to determine the undrained stiffness $C_{i j}^{u}$ 's, and that this $3 \times 3$ matrix has been successfully inverted for the undrained $S_{i j}^{u}$ 's. By taking appropriate sums of (86) and then 
using (84), I find that

$$
\beta_{i} \equiv \sum_{j=1,2,3} S_{i j}^{d}-\frac{1}{3 K_{R}^{g}}=\sum_{j=1,2,3} S_{i j}^{u}-\frac{1}{3 K_{R}^{g}}+B \beta_{i} .
$$

Rearranging (87), I find

$$
(1-B) \beta_{i}=\sum_{j=1,2,3} S_{i j}^{u}-\frac{1}{3 K_{R}^{g}}
$$

Formula (85) determines $B$ exactly in terms of known quantities. Then, Equation (86) plays the same role in the anisotropic results, as (72) did for isotropic porous materials.

Equation (88) is one significant result, but now I want to go farther and show how the same sets of equations can be used in the case when the porosity appears in the form of fluid-filled fractures. In this context, I use the work of Sayers and Kachanov (1991, 1995), as I also do in the main text of this paper. This approach introduces the concept of fractureinfluence parameters $\eta$, measuring the increase in elastic compliance (a weakening effect) due to an oriented crack in some solid elastic background material. The total effect of a set of fractures depends also on the overall fracture density $\rho_{f}$, which is a pure number typically less than unity, shown for example in (4). [See Berryman and Grechka (2006) for examples of the use of numerical experiments to determine the values of the crack parameters $\eta$.] If the background material is the same as the grain material discussed here already, then the concept of fracture influence amounts to formulas of the form:

$$
S_{i j}^{d}=S_{i j}^{g}+\Delta S_{i j}
$$

where the various corrections due to fractures are proportionalities having a form $\Delta S_{i j} \propto$ $\rho_{f} \eta$, or possibly sums of such terms. Typically I find that only two $\eta$ 's are significant at low crack densities, and I call these $\eta_{1}$ and $\eta_{2}$. For purposes of this Appendix, these details do not need to be made explicit. It will be enough to recognize that such contributions give 
rise corrections of the form $\Delta S_{i j}$ as shown in (89). Then, I can show how fluids influence the effects of such fractures.

If I rearrange (86) and then use (89), I have

$$
S_{i j}^{u}=S_{i j}^{d}-\frac{B K_{R}^{d}}{\alpha_{R}} \beta_{i} \beta_{j}=S_{i j}^{g}+\Delta S_{i j}-\frac{B K_{R}^{d}}{\alpha_{R}} \beta_{i} \beta_{j}
$$

Summing this result over both $i$ and $j$, I have:

$$
\frac{1}{K_{R}^{u}}=\frac{1}{K_{R}^{g}}+\sum_{i j} \Delta S_{i j}-\frac{B \alpha_{R}}{K_{R}^{d}}
$$

But I also have

$$
\sum_{i} \beta_{i}=\sum_{i j} \Delta S_{i j}=\frac{\alpha_{R}}{K_{R}^{d}}=\frac{1}{K_{R}^{d}}-\frac{1}{K_{R}^{g}}
$$

So finally, I have the result that

$$
\frac{1}{K_{R}^{u}}=\frac{1}{K_{R}^{g}}+(1-B) \frac{\alpha_{R}}{K_{R}^{d}}=\frac{1}{K_{R}^{g}}+(1-B)\left(\frac{1}{K_{R}^{d}}-\frac{1}{K_{R}^{g}}\right) .
$$

The most important result for our present purposes comes from combining (92) and (93) to obtain:

$$
\frac{1}{K_{R}^{u}}=\frac{1}{K_{R}^{g}}+(1-B) \sum_{i j} \Delta S_{i j}
$$

This equation shows that all the changes introduced via communicating fractures are affected simultaneously in the same way, and this involves a common factor of $(1-B)$ multiplying every fluid-free compliance change $\Delta S_{i j}$. The fluids therefore have the effect of reducing the influence of cracks when they become filled with fluid, since $0 \leq B<1$ always holds for homogeneous porous media. Thus, the general result is an overall strengthening by a factor of $(1-B)$ of the parts of the material saturated with fluid. This anisotropic result is consistent with (and therefore generalizes) a similar result found by the present author for isotropic porous media in an earlier publication (Berryman, 2007). 


\section{APPENDIX B - HETEROGENEOUS POROELASTIC BACKGROUND AND RELATIONSHIP TO BROWN AND KORRINGA RESULTS}

A well-known result in problems concerning heterogeneous poroelastic media is the formula due to Brown and Korringa (1975) found in their equation (32):

$$
S_{i j k l}^{A}-S_{i j k l}^{*}=\frac{\left(S_{i j}^{A}-S_{i j}^{M}\right)\left(S_{k l}^{A}-S_{k l}^{M}\right)}{\left(\kappa_{F}-\kappa_{\phi}\right) \phi+\left(\kappa_{A}-\kappa_{M}\right)}
$$

Although most of my main results are intended to be specific to problems related to fractures, and especially fluid-filled fractures, the general results can nevertheless be put into a form like that of Brown and Korringa (1975). First note that their notation involves both fourth and second rank tensors. My formulation is phrased instead in terms of matrices. The meaning of the various Brown and Korringa (1975) superscripts, when translated into present language, is this: $A \rightarrow d$ (drained), $* \rightarrow u$ (undrained), $M \rightarrow g$ (material or grain), $F \rightarrow f$ (fluid), $\phi \rightarrow \phi$ (porosity). The $\kappa$ 's of Brown and Korringa (1975) are compressibilties, and therefore (for example) $\kappa_{F} \equiv K_{f}^{-1}$ and $\kappa_{\phi} \equiv\left(K_{R}^{\phi}\right)^{-1}$, when translated into bulk moduli.

It is not hard to show that the expression equivalent to that of Brown and Korringa (1975) is given — using current notation for the physical constants from the main text by:

$$
S_{i j k l}^{d}-S_{i j k l}^{u}=\frac{\left(S_{i j}^{d}-S_{i j}^{g}\right) B K_{R}^{d}\left(S_{k l}^{d}-S_{k l}^{g}\right)}{\alpha_{R}}=\left(S_{i j}^{d}-S_{i j}^{g}\right) \gamma^{-1}\left(S_{k l}^{d}-S_{k l}^{g}\right),
$$

where $B$ is Skempton's second coefficient (used extensively in the main text), $\alpha_{R}=1-$

$K_{R}^{d} / K_{R}^{g}$ is the effective stress or Biot-Willis coefficient (Biot and Willis, 1957), $K_{R}^{d}$ is the Reuss average bulk modulus of the drained system. And the very important poroelastic 
coefficient (because it contains all the fluid effects) is

$$
\gamma=\alpha_{R} / B K_{R}^{d}=\left(\kappa_{F}-\kappa_{\phi}\right) \phi+\left(\kappa_{A}-\kappa_{M}\right)=\frac{1}{K_{R}^{d}}-\frac{1}{K_{R}^{g}}+\phi\left(\frac{1}{K_{f}}+\frac{1}{K_{R}^{\phi}}\right)
$$

was introduced earlier in this paper in (24) and is exactly the same expression as (30).

The interested reader might want to verify (for the sake of personal knowledge and/or satisfaction) that (96) is in fact equivalent to (49) [in compliance notation], and also to (46) [in stiffness notation]. 


\section{LIST OF TABLES}

1 Compliance matrix correction values for the vertical fracture model considered in Eq. 5, which are also valid for the specific limit of Eq. 8, as a special case of the general

result. $\mathcal{A}_{f}, \mathcal{B}_{f}$, and $\rho_{f}$ are all measures of fracture density, pertinent to particular components (as shown) of the compliance matrix modifiers in $\Delta \mathbf{S}$.

2 Fracture-influence parameters [see Table 1 for usage and Eqs. (6) and (7) for their NIA definitions] in a model reservoir having isotropic background with Poisson's ratio $\nu=0.4375, V_{p}=3.0 \mathrm{~km} / \mathrm{s}$, and $V_{s}=1.0 \mathrm{~km} / \mathrm{s}$. This model assumes an isotropic background, which is treated here as an especially simple case of VTI. Note that as a general rule $0 \leq\left|\eta_{1} / \eta_{2}\right| \leq 0.05$

3 Constant $V_{s v}$ wave speeds in the $\left[x_{1}-x_{3}\right]$ plane found for various values of the angle $\phi$ between fracture planes, and for the fixed value of fracture density $\rho_{f}=0.20$.

4 Values of Skempton's coefficient $B$ (dimensionless) for various common earth materials (Wang, 2000). Note that $B$ is a function of the state of stress of the porous material, so these are nominal values.

5 Symbols 


\section{LIST OF FIGURES}

1 Illustrating the concept of the two nonintersecting and nonparallel vertical fracture sets.

2 A set of three examples of compressional wave speed $(a, b, c)$ for equal fracture densities $\rho_{a}=\rho_{b}=\rho_{f} / 2$. Angles between fracture planes are, respectively, $\phi=30^{\circ}, 60^{\circ}$, and $90^{\circ}$.

3 A set of three examples of $\mathrm{SH}$-wave speed $(\mathrm{a}, \mathrm{b}, \mathrm{c})$ for equal fracture densities $\rho_{a}=$ $\rho_{b}=\rho_{f} / 2$. Angles between fracture planes are, respectively, $\phi=30^{\circ}, 60^{\circ}$, and $90^{\circ}$.

4 A set of three examples of $S V$-shear wave speed for equal fracture densities $\rho_{a}=$ $\rho_{b}=\rho_{f} / 2$ : angles between fracture planes are $\phi=30^{\circ}, 60^{\circ}$, and $90^{\circ}$. Constant values seen here are also shown in Table 3. 


\begin{tabular}{|l}
\hline$\Delta S_{11}=\mathcal{A}_{f}\left(\eta_{1}+\eta_{2}\right)$ \\
\hline$\Delta S_{22}=\mathcal{B}_{f}\left(\eta_{1}+\eta_{2}\right)$ \\
\hline$\Delta S_{33}=0$ \\
\hline$\Delta S_{12}=\rho_{f} \eta_{1}$ \\
\hline$\Delta S_{13}=\mathcal{A}_{f} \eta_{1} / 2$ \\
\hline$\Delta S_{23}=\mathcal{B}_{f} \eta_{1} / 2$ \\
\hline$\Delta S_{44}=\mathcal{B}_{f} \eta_{2}$ \\
\hline$\Delta S_{55}=\mathcal{A}_{f} \eta_{2}$ \\
\hline$\Delta S_{66}=2 \rho_{f} \eta_{2}$ \\
\hline
\end{tabular}

Table 1: Compliance matrix correction values for the vertical fracture model considered in Eq. 5, which are also valid for the specific limit of Eq. 8, as a special case of the general result. $\mathcal{A}_{f}, \mathcal{B}_{f}$, and $\rho_{f}$ are all measures of fracture density, pertinent to particular components (as shown) of the compliance matrix modifiers in $\Delta \mathbf{S}$. 


\begin{tabular}{|c|c|}
\hline Fracture parameter & $\mathrm{GPa}^{-1}$ \\
\hline$\eta_{1}$ & -0.0192 \\
\hline$\eta_{2}$ & 0.3944 \\
\hline
\end{tabular}

Table 2: Fracture-influence parameters [see Table 1 for usage and Eqs. (6) and (7) for their NIA definitions] in a model reservoir having isotropic background with Poisson's ratio $\nu=$ $0.4375, V_{p}=3.0 \mathrm{~km} / \mathrm{s}$, and $V_{s}=1.0 \mathrm{~km} / \mathrm{s}$. This model assumes an isotropic background, which is treated here as an especially simple case of VTI. Note that as a general rule $0 \leq\left|\eta_{1} / \eta_{2}\right| \leq 0.05$ 


\begin{tabular}{|c|c|}
\hline$\phi$ & $V_{s v}(\mathrm{~km} / \mathrm{s})$ \\
\hline $0^{\circ}$ & 0.8602 \\
\hline $30^{\circ}$ & 0.8678 \\
\hline $45^{\circ}$ & 0.8771 \\
\hline $60^{\circ}$ & 0.8896 \\
\hline $90^{\circ}$ & 0.9222 \\
\hline
\end{tabular}

Table 3: Constant $V_{s v}$ wave speeds in the $\left[x_{1}-x_{3}\right]$ plane found for various values of the angle $\phi$ between fracture planes, and for the fixed value of fracture density $\rho_{f}=0.20$. 


\begin{tabular}{|l|l|}
\hline Rock & $B$ \\
\hline \hline Berea sandstone & 0.62 \\
\hline Boise sandstone & 0.50 \\
\hline Kayenta sandstone & 0.67 \\
\hline Indiana limestone & 0.46 \\
\hline Tennessee marble & 0.51 \\
\hline Westerly granite & 0.85 \\
\hline
\end{tabular}

Table 4: Values of Skempton's coefficient $B$ (dimensionless) for various common earth materials (Wang, 2000). Note that $B$ is a function of the state of stress of the porous material, so these are nominal values. 


\begin{tabular}{|c|c|}
\hline$e_{i j}$ & volume strain \\
\hline$k_{1}, k_{2}, k_{3}$ & wavenumbers for directions $x, y, z$ \\
\hline$p_{c}, p_{f}$ & confining and fluid pressures \\
\hline$A, B$ & Skempton's first and second coefficients \\
\hline$C_{i j}$ & elastic stiffness matrix \\
\hline$E_{0}, G_{0}, K_{0}$ & Young's, shear, and bulk moduli of host medium \\
\hline$K_{R}^{g}, K_{R}^{\phi}, K_{R}^{d}, K_{R}^{u}$ & bulk moduli for grains, pores, drained \& undrained systems \\
\hline$K_{f}, K_{\text {susp }}$ & bulk moduli for pore-fluid and fluid-grain suspension \\
\hline$S_{i j}=S_{i j}^{d}$ & elastic (drained) compliance matrix \\
\hline$S_{i j}^{u}$ & poroelastic (undrained) compliance matrix \\
\hline$V_{ \pm}$ & wave speeds for $P$ - and $S V$-waves \\
\hline $\mathcal{A}_{f}, \mathcal{B}_{f}$ & fracture densities for vertically fractured systems \\
\hline$\beta_{1}, \beta_{2}, \beta_{3}$ & off-diagonal poroelastic compliances \\
\hline$\gamma$ & diagonal poroelastic compliance \\
\hline$\epsilon, \delta$ & two of Thomsen's weak anisotropy parameters \\
\hline$\zeta$ & increment of fluid content \\
\hline$\eta_{1}, \eta_{2}$ & two fracture-influence compliance parameters \\
\hline$\nu_{0}$ & Poisson's ratio of host medium \\
\hline$\rho_{0}$ & inertial density \\
\hline$\rho_{f}$ & fracture density \\
\hline$\sigma_{i j}$ & external stress matrix/tensor \\
\hline$\phi$ & porosity \\
\hline
\end{tabular}

Table 5: Symbols 


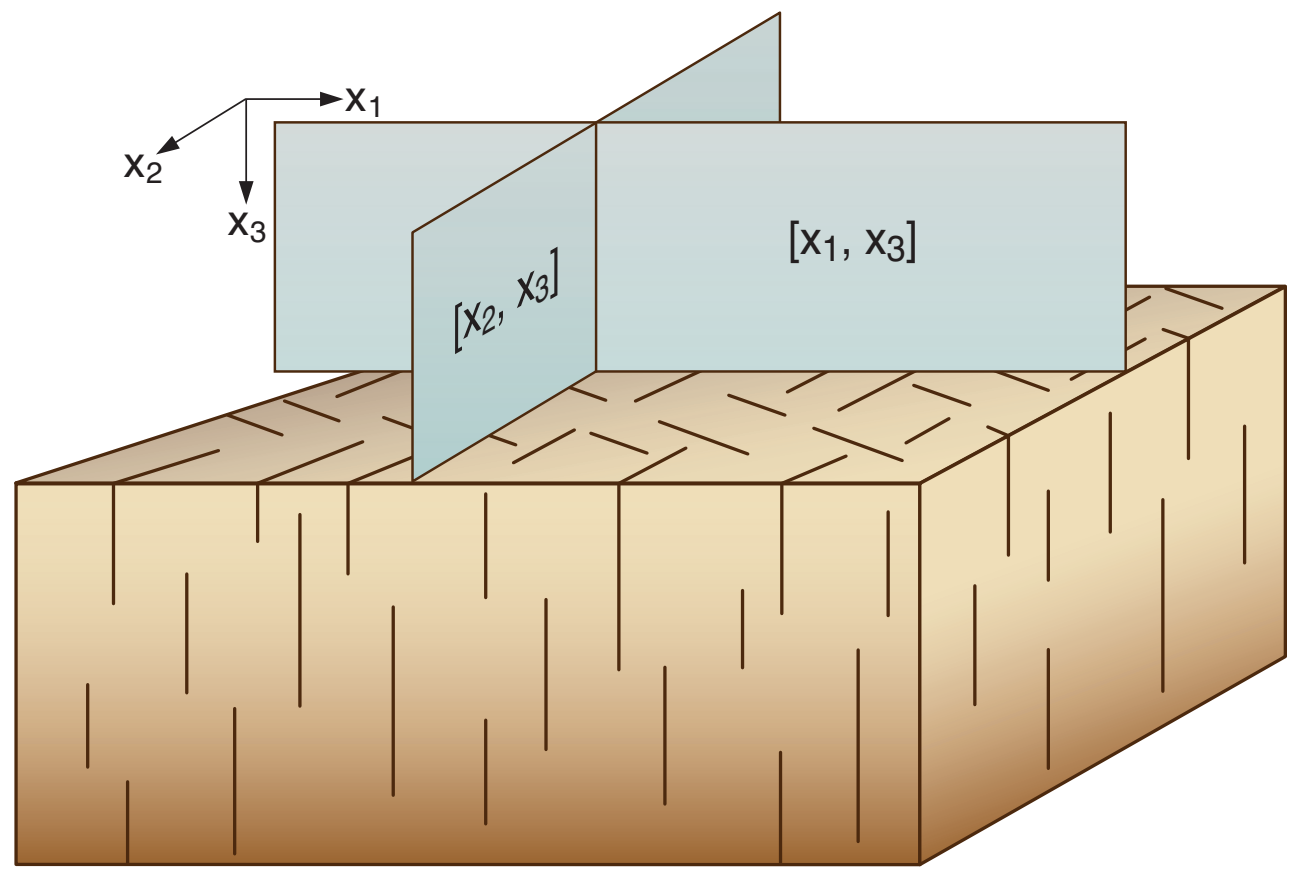

Figure 1: Illustrating the concept of the two nonintersecting and nonparallel vertical fracture sets.

\section{Berryman -}




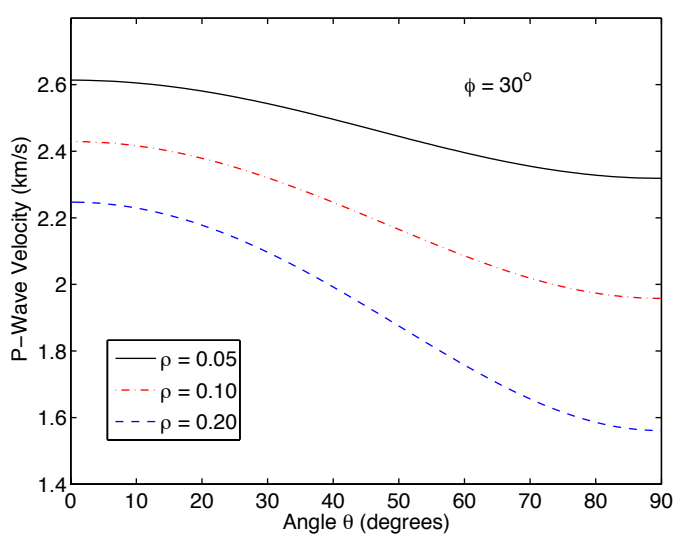

(a)

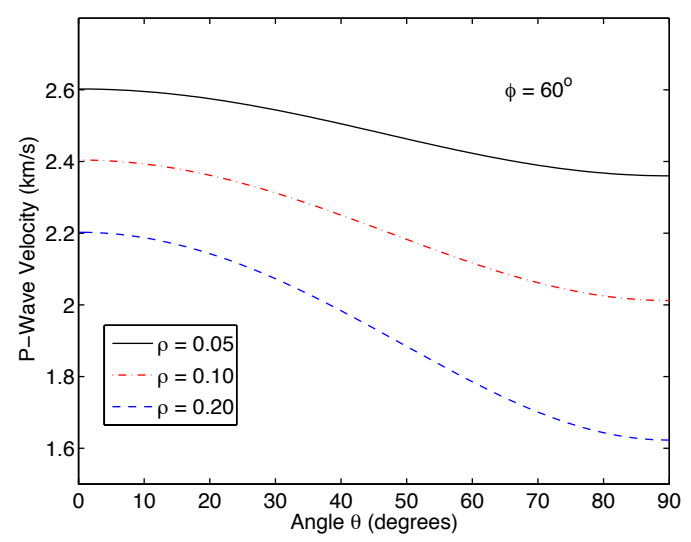

(b)

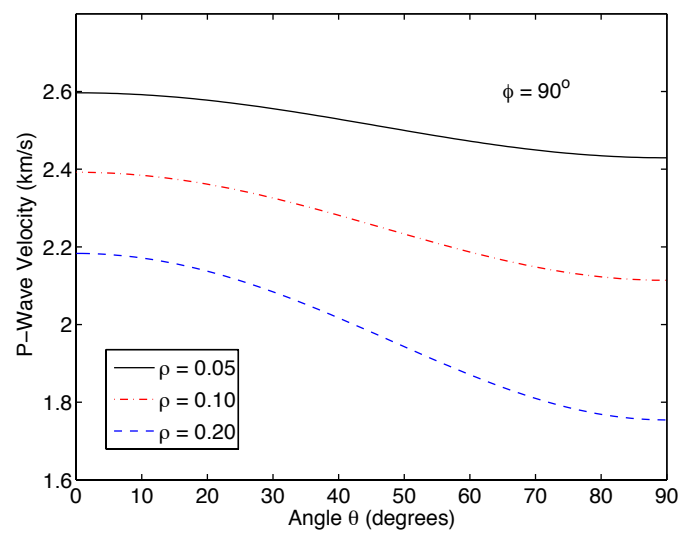

(c)

Figure 2: A set of three examples of compressional wave speed $(a, b, c)$ for equal fracture densities $\rho_{a}=\rho_{b}=\rho_{f} / 2$. Angles between fracture planes are, respectively, $\phi=30^{\circ}, 60^{\circ}$, and $90^{\circ}$.

\section{Berryman -}



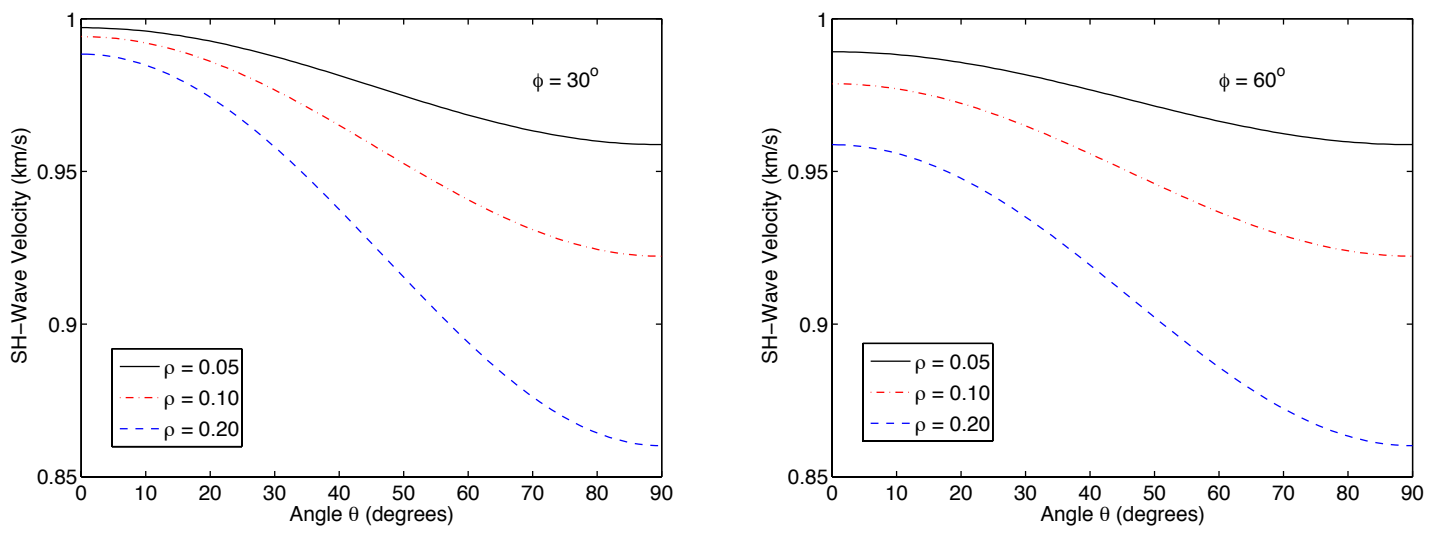

(a)

(b)

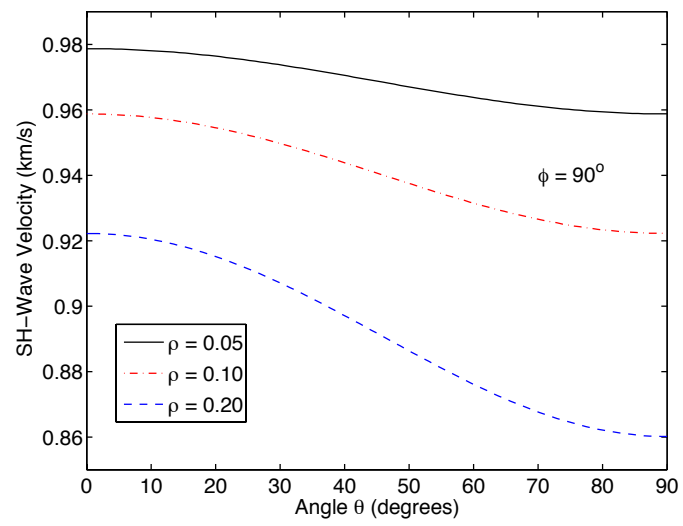

(c)

Figure 3: A set of three examples of $\mathrm{SH}$-wave speed $(\mathrm{a}, \mathrm{b}, \mathrm{c})$ for equal fracture densities $\rho_{a}=\rho_{b}=\rho_{f} / 2$. Angles between fracture planes are, respectively, $\phi=30^{\circ}, 60^{\circ}$, and $90^{\circ}$.

\section{Berryman -}




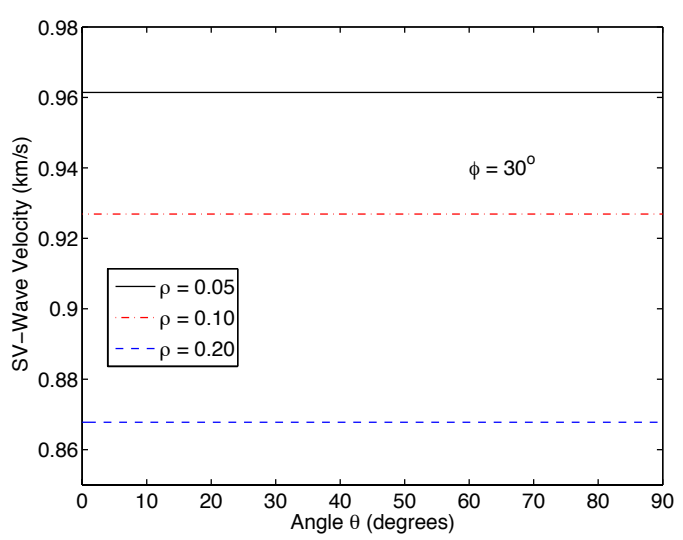

(a)

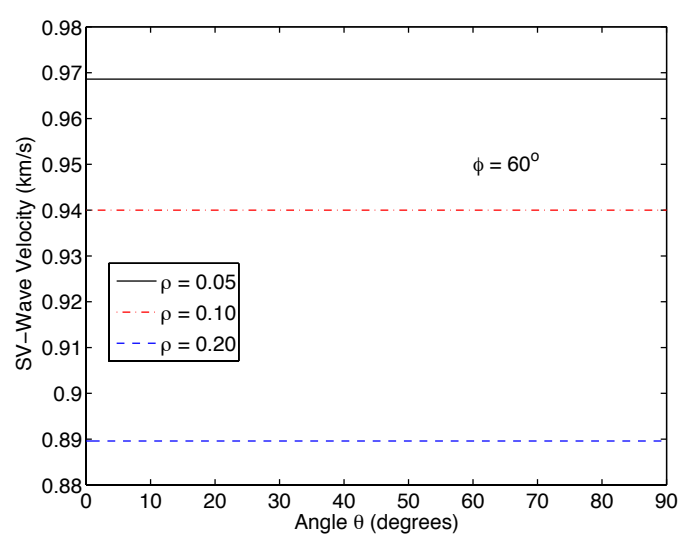

(b)

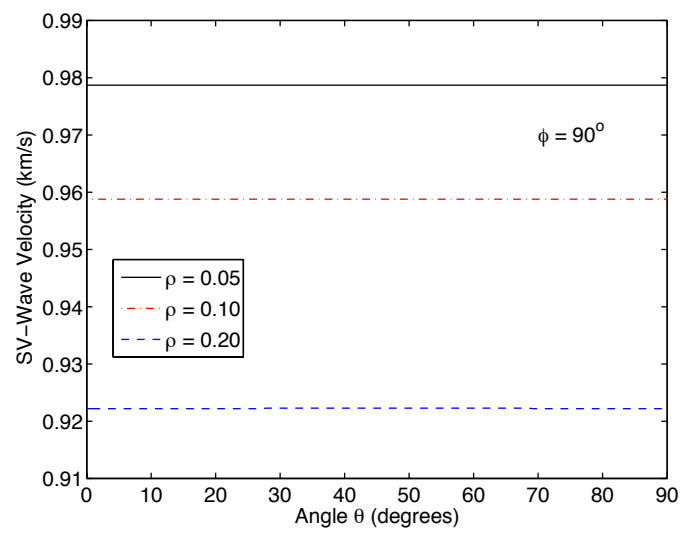

(c)

Figure 4: A set of three examples of $S V$-shear wave speed for equal fracture densities $\rho_{a}=\rho_{b}=\rho_{f} / 2$ : angles between fracture planes are $\phi=30^{\circ}, 60^{\circ}$, and $90^{\circ}$. Constant values seen here are also shown in Table 3.

\section{Berryman -}




\section{DISCLAIMER}

This document was prepared as an account of work sponsored by the United States Government. While this document is believed to contain correct information, neither the United States Government nor any agency thereof, nor The Regents of the University of California, nor any of their employees, makes any warranty, express or implied, or assumes any legal responsibility for the accuracy, completeness, or usefulness of any information, apparatus, product, or process disclosed, or represents that its use would not infringe privately owned rights. Reference herein to any specific commercial product, process, or service by its trade name, trademark, manufacturer, or otherwise, does not necessarily constitute or imply its endorsement, recommendation, or favoring by the United States Government or any agency thereof, or The Regents of the University of California. The views and opinions of authors expressed herein do not necessarily state or reflect those of the United States Government or any agency thereof or The Regents of the University of California.

Ernest Orlando Lawrence Berkeley National Laboratory is an equal opportunity employer. 